\section{1}

2

3

\section{4}

5

6

7

Corresponding author: Miguel-Godinho.FERREIRA@unice.fr Godinho Ferreira ${ }^{1,2}$

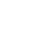
U1081 Université Côte d'Azur, 06107 Nice, France.

${ }^{2}$ Instituto Gulbenkian de Ciência, 2780-156 Oeiras, Portugal. alternatives (CEA), Université Côte d'Azur, 06107 Nice, France Cancer Medicine, Sun Yat-sen University Cancer Center, Guangzhou, China.

\title{
Telomere elongation in the gut extends zebrafish lifespan
}

Mounir El Maï ${ }^{1,2}$, Jean-Marie Guigonis ${ }^{3}$, Thierry Pourchet ${ }^{3}$, Da Kang ${ }^{4}$, Jia-Xing Yue $^{4}$, Miguel

${ }^{1}$ Institute for Research on Cancer and Aging of Nice (IRCAN), CNRS UMR7284 INSERM

${ }^{3}$ Laboratory Transporter in Imaging and Radiotherapy in Oncology (TIRO), Institut des sciences du vivant Frederic Joliot, Commissariat à l'Energie Atomique et aux Energies ${ }^{4}$ State Key Laboratory of Oncology in South China, Collaborative Innovation Center for

31 Keywords: Aging, Telomerase, Gut, Tissue-specific, Systemic effects, Zebrafish 


\section{ABSTRACT: (150 words 1116 char)}

Telomere shortening is a hallmark of aging and is counteracted by telomerase. The gut is one of the earliest organs to exhibit short telomeres and tissue dysfunction during normal zebrafish aging. This is recapitulated in prematurely aged telomerase mutants (tert-/-). Here, we show that gut-specific telomerase activity in tert-/- zebrafish prevents premature aging. Induction of telomerase rescues gut senescence and low cell proliferation to wild-type levels, while restoring gut tissue integrity, inflammation, and age-dependent gut microbiota dysbiosis. Remarkably, averting gut dysfunction results in a systemic beneficial impact. Gut-specific telomerase activity rescues premature aging markers in remote organs, such as the reproductive (testes) and hematopoietic (kidney marrow) systems. Functionally, it also rescues agedependent loss of male fertility and testes atrophy. Finally, we show that gut-specific telomerase activity increases the lifespan of telomerase mutants. Our work demonstrates that delaying telomere shortening in the gut is sufficient to systemically counteract aging in zebrafish.

\section{INTRODUCTION:}

The discovery that lifespan can be genetically extended in C. elegans, initiated a new era of research aiming to define interventions that promote lifespan and healthspan extension ${ }^{1}$. Since then, health and lifespan improvements have been achieved by modulating hallmarks of aging and provided promising therapeutical targets for healthy aging ${ }^{2}$. For example, reverting age-related deregulation of nutrient-sensing mechanisms by interventions such as caloric restriction or rapamycin (mTOR inhibitor) treatment was observed to increase lifespan in several species $^{3,4}$. Similarly, genetic or pharmacologic removal of senescent cells can delay ageassociated defects resulting in lifespan extension in mice e.6. $^{5,6}$.

Telomere shortening and damage are major determinants contributing to aging ${ }^{2}$. Telomeres protect chromosome ends from degradation and recognition by DNA damage response pathways ${ }^{7}$. Due to the "end-replication problem", telomeres gradually shorten with each round of cell division ${ }^{7}$. When telomeres become critically short, DNA damage responses are triggered and culminate in cell cycle arrest and, eventually, replicative senescence ${ }^{8,9}$. Consequently, reduced proliferation and accumulation of senescent cells result in loss of tissue integrity $^{2}$. Telomere shortening is counteracted by a specific reverse transcriptase termed telomerase. Telomerase is a multi-subunit ribonucleoprotein, with TERT being its main catalytic component. TERT expression is limited mainly to stem or progenitor cells ${ }^{10,11}$. However, telomerase activity is insufficient to prevent telomere attrition during aging ${ }^{11}$.

Telomeropathy patients carry mutations in telomerase or telomere maintenance protein genes, which lead to premature shortening of telomeres and short life expectancy ${ }^{12,13}$. Similarly, telomerase deficiency in tert-/- zebrafish accelerates telomere shortening, leading to premature aging phenotypes and reduced lifespan already in the first generation ${ }^{14-16}$. The majority of tissue dysfunction events described during natural zebrafish aging are anticipated during tert-/zebrafish aging ${ }^{14-16}$. The gut is one of the first organs to exhibit DNA damage associated with short telomeres, reduced cell proliferation, senescence and functional defects not only in natural aging but also throughout tert-/- life ${ }^{14,17}$. Importantly, telomere shortening accelerates cellular and functional defects in the gut at a time when other organs remain clear of tissue 
dysfunction ${ }^{14}$. As in zebrafish, the human gastrointestinal system is one of the organs with the fastest rate of telomere shortening ${ }^{18}$. Telomeropathy patients are often associated with gastrointestinal syndromes ${ }^{19,20}$ and increased telomere shortening was observed in the intestinal epithelium of inflammatory bowel disease patients ${ }^{21,22}$. Therefore, gut homeostasis is intricately connected to telomere length.

A crucial role of gut homeostasis has been described for organism health. Loss of gut permeability is involved in several disorders such as inflammatory bowel disease, diabetes, chronic heart failure and even Parkinson disease ${ }^{4}$. Modification of gut microbiota content (dysbiosis) is associated to aging ${ }^{23,24}$ and is involved in age-related systemic inflammation ${ }^{25}$. Even though weakening of the intestinal barrier is as major feature of gut aging ${ }^{4}$, it remains unclear whether gut aging influences overall organismal aging. Inflammatory and SASP (senescence-associated secretory phenotype) factors chronically emanating from intestinal epithelium with critically short telomeres may impact systemic homeostasis.

Considering that the gut is one of the first organs exhibiting telomere-dependent aging, we anticipated that delaying gut aging would be beneficial for the entire organism. Here, we present a novel vertebrate model aimed at investigating the impact of telomere-dependent gut aging on the entire organism. Using a zebrafish line containing a Cre-inducible and gut-specific tert transgene, we show that enterocyte-specific telomerase activity in tert-/- fish is sufficient to delay gut aging. Counteracting gut aging improves health of the entire organism, reverting gut microbiota dysbiosis and aging phenotypes in the reproductive and hematopoietic system of tert-/- zebrafish. Finally, we show that the most relevant systemic effect of gut-specific telomerase activity is lifespan extension. Thus, gut telomere-dependent aging controls aging of the entire organism.

\section{RESULTS:}

\section{Tissue-specific telomerase activity rescues gut aging}

As in humans, the zebrafish gut is one of the organs to exhibit fast telomere length decline $^{14,18}$. To investigate how telomere-dependent gut aging impacts the organism, we generated a Cre-inducible zebrafish transgenic line with gut-specific tert expression. This line contains an enterocyte-specific Fabp-2 promoter $^{26}$ upstream of a lox-STOP-lox cassette followed by zebrafish tert cDNA in an tert + - genetic background (Figure 1A). After crossing this line with tert+/- fish, we induced the tert transgene expression by micro-injection of Cre mRNA in one-cell stage embryos. Mock injected fish were used as controls for injection and transgene genomic position effects. This experimental set up provided sibling fish that were either tert-/- containing the full construct ("tert-/- No Cre"; from mock injected embryos), tert/- expressing tert transgene ("tert-/- +Cre", from Cre mRNA injected embryos), and tert+/+ containing the full construct ("WT"; from mock injected embryos). Thus, this line allows us to investigate the effects of telomerase activity specifically in the gut of telomerase deficient fish. While we did not detect expression in mock injected fish, Cre-mediated removal of the STOP cassette triggered the transcription of tert transgene in gut tissue (Figure 1B; left panel). This led to $\sim 5$-fold enrichment of total tert mRNA (endogenous and transgene tert mRNA) in 
Figure 1

A.

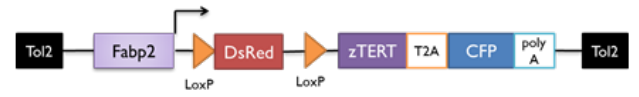

B.

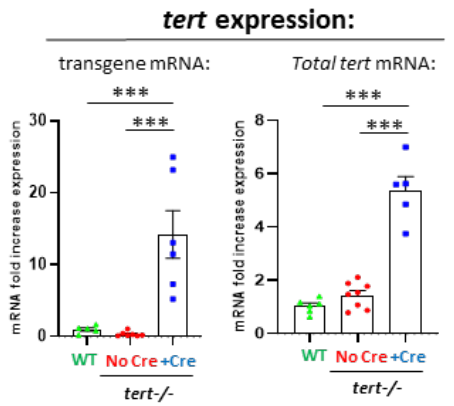

C.

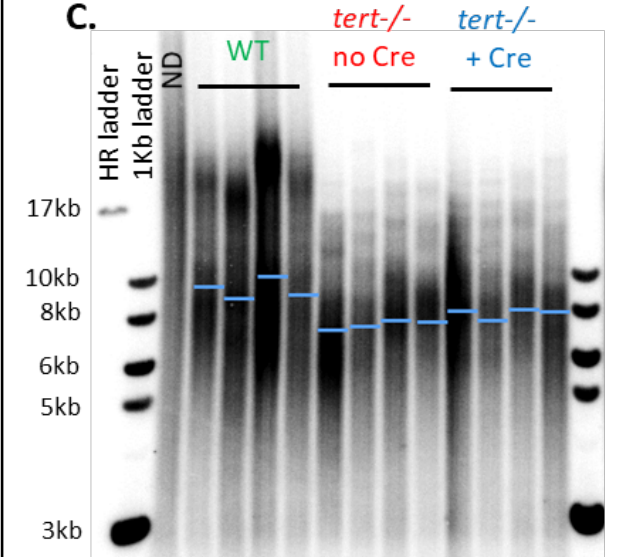

D.

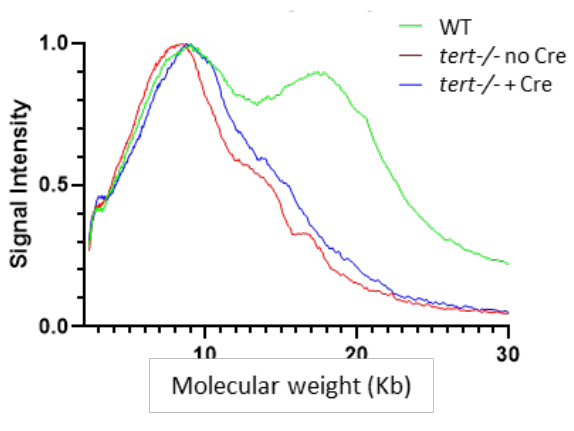

E.

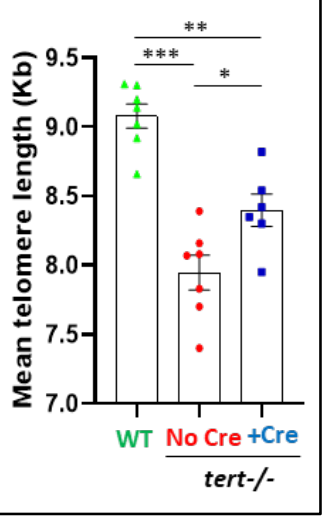

\section{Figure 1: Cre-mediated tert expression extend telomere length in tert-/- gut tissue.}

A. Schematic representation of the transgene allowing for a Cre inducible and enterocyte specific expression of tert mRNA. We created a zebrafish line containing an enterocyte-specific promoter (Fabp2: fatty acid binding protein 2) controlling DsRed gene expression flanked by two LoxP sites. Tert-T2A-CFP polycistronic gene was added downstream of the second LoxP site. B. RT-qPCR analysis of tert transgene mRNA and total tert mRNA (endogenous + transgene) expression in 9-month-old gut extracts. RT-qPCR graphs are representing mean \pm SEM mRNA fold increase after normalization by rps 11 gene expression levels ( $\mathrm{N}=5-8$; *** p-value $<0.001$, using oneway ANOVA and post-hoc Tuckey tests). Cre mRNA injection at one cell-stage embryos induces the transcription of tert transgene mRNA. C. Representative images of telomere restriction fragment (TRF) analysis by Southern Blot of genomic DNA extracted from 9-month-old gut samples and quantifications of mean telomere length (blue bars). D. TRF mean densitometry curves $(\mathrm{N}=6-7)$. E. Quantification of mean telomere length analyzed by TRF. Cre-mediated and enterocyte specific tert expression elongated telomere length in gut of tert-/- fish. Data are represented as mean + - SEM $\left(\mathrm{N}=6-7 ; * \mathrm{p}\right.$-value $<0.05 ;{ }^{* *} \mathrm{p}$-value $<0.01$, ${ }^{* * *} \mathrm{p}$-value $<0.001$, using one-way ANOVA and post-hoc Tuckey tests).

the gut of tert-/- +Cre fish when compared to mock injected control tissues (tert-/- No Cre and WT) (Figure 1B; right panel). To test whether expression of the tert transgene is sufficient to prevent telomere shortening, we performed Telomere Restriction Fragment (TRF) analysis on gut samples of 9-month-old fish. As previously shown, we observed that the range of telomere length in the gut of WT fish exhibits a bimodal pattern (Figure 1C-D) ${ }^{14,15}$. This pattern reflects the differences in telomere length between cell types. Telomere length of WT blood cells is longer $(\sim 19 \mathrm{~kb})$ than other tissues ( $\sim 9 \mathrm{~kb}$ ) leading to a densitometry pattern with two peaks ${ }^{14,15}$. Reflecting the requirement of telomerase activity to sustain long telomeres in blood cells, 
telomere length of tert-/- blood cells is drastically reduced compared to WT (as seen by the loss of the longer telomere peak, Figure 1D) $)^{14,15}$. Consequently, tert-/- No Cre presented a unimodal TRF pattern in the intestinal tissue. Even though expression of tert cDNA driven by the Fabp2 promoter did not fully restore telomere length to WT levels, induction of the tert transgene is sufficient to elongate telomeres in whole gut tissues of tert-/- +Cre fish $(7.9 \mathrm{~kb}$ to $8.4 \mathrm{~kb}, \mathrm{~N}=6$ 7; $<<0.05$; Figure 1E). Like tert-/- No Cre fish, tert-/- +Cre fish lacked the higher molecular weight telomere peak, indicating that the tert transgene is not expressed in blood cells.

Described as a hallmark of aging, telomere erosion has been proposed as a "molecular clock" defining the number of cell divisions before cell cycle arrest, cell death or replicative senescence ${ }^{2}$. Reduced cell proliferation and accumulation of senescent cells limits homeostatic regeneration and, consequently, causes loss of tissue integrity. In agreement, accelerated telomere shortening in tert-/- fish results in premature aging phenotypes ${ }^{14}$. In order to test whether tert transgene expression in the gut of tert-/- fish rescues local aging defects, we analysed the gut of 9-month-old fish. As previously reported, compared to WT fish, the gut of tert-/- No Cre fish showed a reduced proliferation rate ${ }^{14,15}$. Notably, enterocyte-specific telomerase activity rescued the proliferative capacity of this organ to WT levels (Figure 2A). Similarly, SA- $\beta$-galactosidase assays and transcription levels of the senescence-associated genes $p 15 / 16$ and $p 21$ revealed that telomerase activity reduces cell senescence to WT levels (Figure 2B-D). We previously described a cell fate switch from apoptosis to senescence in old tert-/- where senescence becomes predominant ${ }^{17}$. At that age, onset of apoptosis becomes indistinguishable between WT and tert-/. Consistently, we detected no difference in number of apoptotic cells in the intestinal epithelium at 9 months of age between WT, tert-/- No Cre and tert-/- +Cre fish (Supplementary figure 1A).

These cellular defects observed in tert-/- fish impact tissue integrity ${ }^{14,15,17}$. We observed that 9-month-old tert-/- No Cre fish exhibit morphological tissue defects with thickening of the lamina propria as compared to WT (Figure 2E-F). Loss of intestinal barrier integrity leads to activation of the YAP (Yes-associated protein) transcription factor responsible for tissue regeneration ${ }^{27,28}$. Consistent with loss of gut integrity, expression of the YAP-target genes cyr61 and ctgf are increased in tert-/- No Cre fish compared to WT (Figure 2G-H). Likewise, claudin-2 mRNA levels are higher in tert-/- No Cre compared to WT (Figure 2I). Increased gene expression of the tight-junction protein Claudin-2 occurs during primate aging and enhances in vivo intestinal permeability ${ }^{29,30}$. Strikingly, all these phenotypes were rescued in tert-/- +Cre fish (Figure 2E-I). As a consequence of loss of intestinal integrity, we observed higher inflammation in the intestinal epithelium of the tert-/- No Cre fish as compared to WT. We detected an increased infiltration of eosinophiles (Figure 2J) and neutrophils (Figure 2K) in the gut of tert-/- No Cre fish. In line with a rescue of intestinal integrity, the number of these myeloid immune cells was similar to WT in tert-/- +Cre fish. Although not significant, gutspecific telomerase activity also ameliorates increased il6 gene expression observed in tert-/No Cre compared to WT (Figure 2L). Similarly, while no difference was detected in tnfa mRNA levels between tert-/- No Cre and WT, the expression of this inflammation-related gene is reduced in tert-/- +Cre fish (Figure $2 \mathrm{M}$ ). 
Figure 2
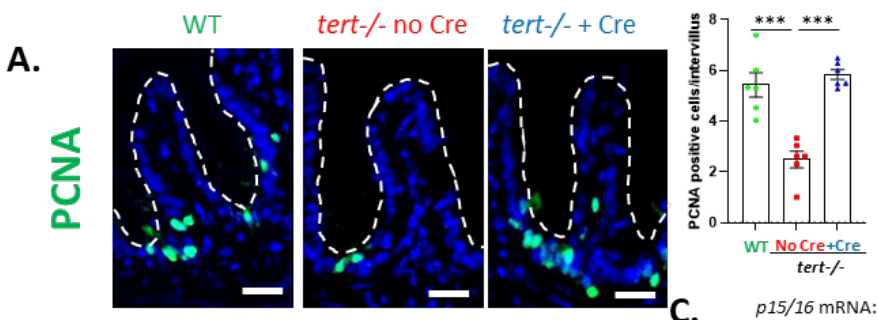

B.
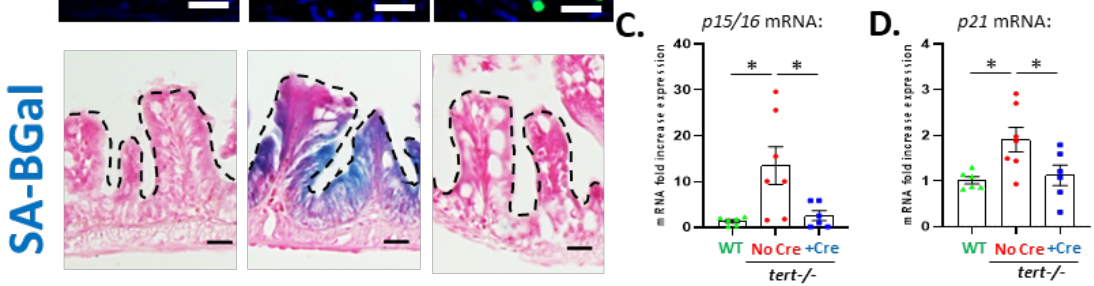

E.
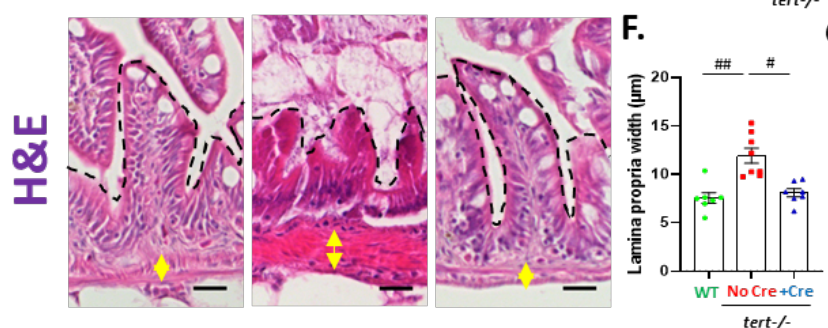

G. cyr61 mRNA:

H. $\operatorname{ctg} f \mathrm{mRNA}$ :

I. claudin-2 mRNA:

J.
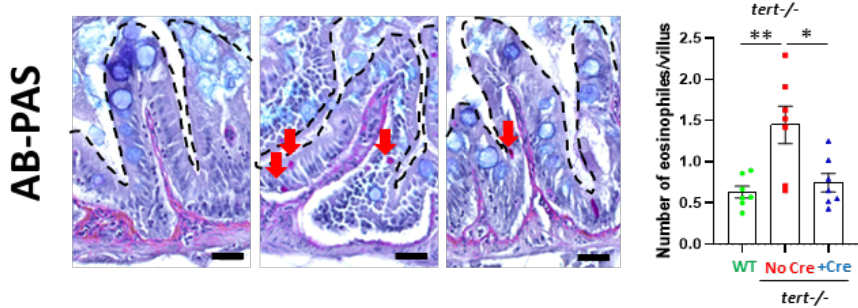

K.
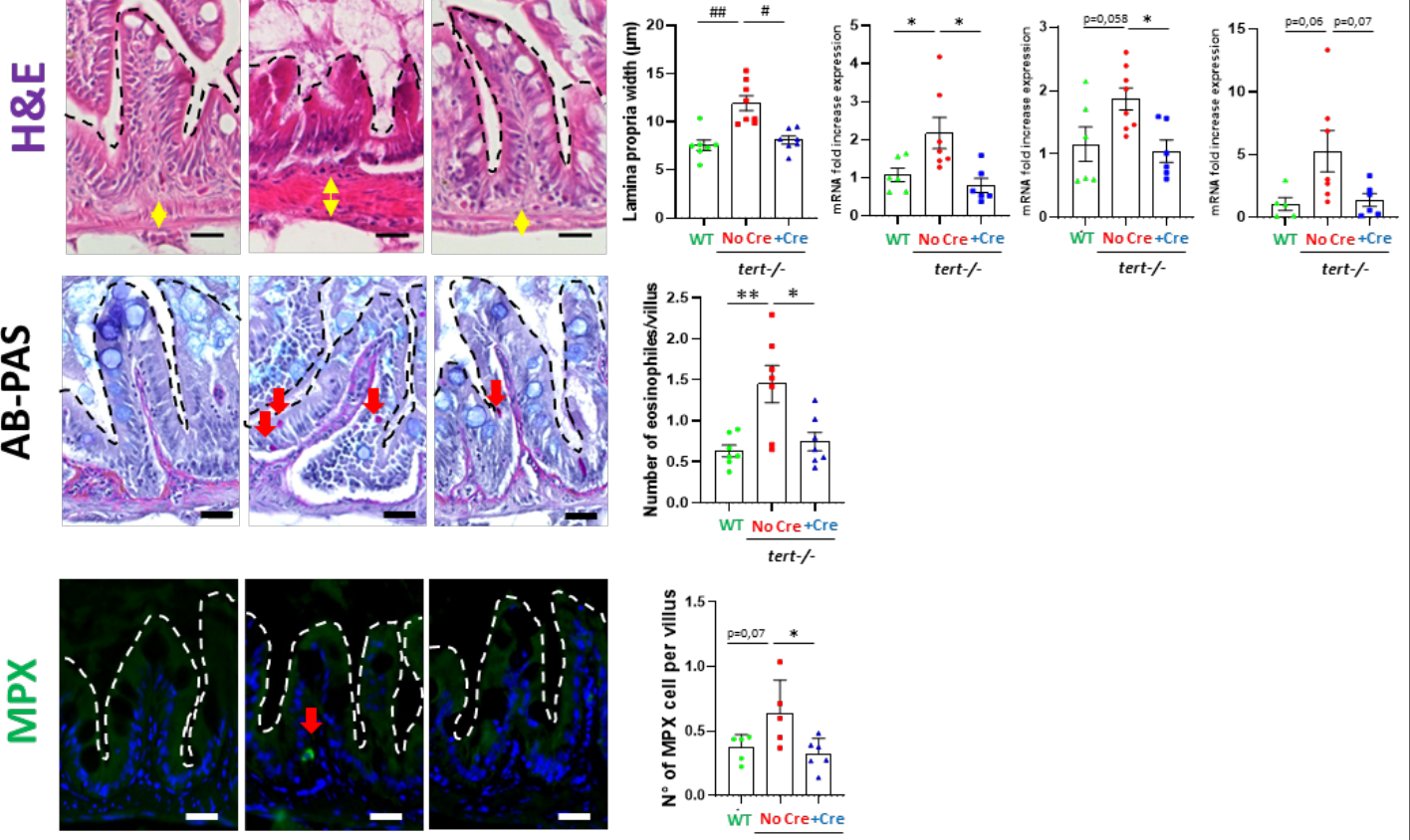

L.

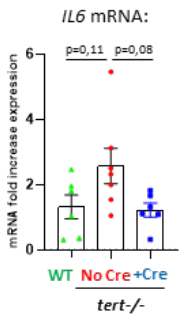

M. tnfamRnA:

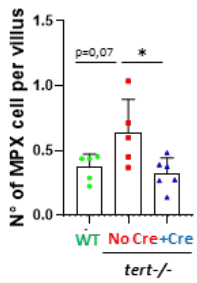

Figure 2: Telomerase reactivation rescues gut aging phenotypes.

A. Representative immunofluorescence images of proliferation staining (PCNA marker; left panel) and quantification (right panel) in 9-month-old gut tissues. B. Representative image of SA- $\beta$-Gal staining of 9-monthold gut cryosection. C-D. RT-qPCR analysis of senescence-associated genes $p 15 / 16$ (C.) and $p 21$ (D.) expression in 9-month-old gut samples. Telomere elongation in gut of tert-/- +Cre fish rescues both proliferation and senescence to WT levels compared to tert-/- No Cre fish. E. Representative hematoxylin and eosin-stained sections of gut from 9-month-old fish (yellow arrows delineate lamina propria width quantified in F.). F. Quantification of lamina propria width measured on histology images of 9-month-old fish gut. G-H. RT-qPCR analysis of YAP target genes cyr61 (G.) and $\operatorname{ctg} f(\mathbf{H}$.) expression in 9-month-old gut samples. I. RT-qPCR analysis of the junction 
protein associated gene claudin-2 expression in 9-month-old gut samples. J. Representative AB-PAS staining images of 9-month-old fish gut (left panel). Number of pink-staining eosinophile cells (red arrows) are quantified in the right panel. K. Representative immunofluorescence images of neutrophil staining (MPX marker; left panel) and quantification (right panel) in 9-month-old gut tissues. L-M. RT-qPCR analysis of inflammation-associated genes il6 (C.) and tnfa (D.) expression in 9-month-old gut samples. Telomere elongation in gut of tert-/- +Cre fish rescues gut integrity and consequent gut inflammation to WT levels compared to tert-/- No Cre fish. Scale bar: $20 \mu \mathrm{m}$. Dashed lines delineate gut villi. All data are represented as mean $+/-$ SEM $(\mathrm{N}=6-8$ per condition; * $\mathrm{p}-$ value $<0.05 ; * *$ p-value $<0.01,{ }^{* * *}$ p-value $<0.001$, using one-way ANOVA and post-hoc Tuckey tests; \# pvalue $<0.05$; \#\# p-value $<0.01$, \#\#\# p-value $<0.001$, using Kruskal-Wallis and post-hoc Dunn's tests). All RT-qPCR graphs are representing mean \pm SEM mRNA fold increase after normalization by rps 11 gene expression levels.

By comparing the expression profiles of whole gut tissues using RNA sequencing, we observed a distinguishable transcriptomic signature of tert-/- No Cre, while WT and tert-/- +Cre samples clustered together (Supplementary figure 2A). GO term analyses showed that, compared to tert-/- No Cre, both WT and tert-/- +Cre are enriched in gene expression related to cell cycle and ATP production in addition to reduced transcription of genes related to morphogenesis (Supplementary figure 2B-E). Accordingly, KEGG GSEA analyses showed an increase in ribosome and oxidative phosphorylation in these two groups compared to tert-/- No Cre, and a decrease of phagosome, cytokine signalling and neuroactive ligand receptor interaction which encompasses arachidonic inflammatory pathway (Supplementary figure 2F$\mathrm{G})$. In line with the previous results, these transcription profiles confirmed that telomerase activity rescued cell proliferation defects, loss of tissue integrity and inflammation seen in gut of tert-/- No Cre fish.

\section{Local effects: Gut-specific telomerase activity rescues gut microbiota dysbiosis}

Gut microbiota dysbiosis is associated with a dysfunctional intestinal barrier and is proposed to generate a feed-forward loop involving gut permeability, inflammation and dysbiosis in aging ${ }^{25,31}$. However, it remains unclear whether delaying gut aging counteracts gut microbiota (GM) dysbiosis. To investigate if telomerase activity in the gut of tert-/- fish ameliorates gut dysbiosis, we performed high-throughput sequencing of the V3-4 region of 16S-rDNA of 9-month-old zebrafish gut. Similar to what is described for human aging ${ }^{23,32}$, we observed diminished microbial diversity in tert-/- No Cre when compared to WT controls. Both alpha (within samples) and beta (within groups) analyses showed lower diversity in tert-/- No Cre individuals compared to WT and tert-/- +Cre fish (Figure 3A-B). According to a reduced beta-diversity, using principal coordinates analysis (PCoA), we observed a clustering of tert-/No Cre samples while WT and tert-/- +Cre samples were more dispersed (Figure 3C).

Relative abundance analysis of bacterial taxonomic units (BTUs) at the class level revealed an overall alteration of GM composition in tert-/- No Cre fish compared to WT that was recovered by tert transgene expression (Figure 3D). At the class level, we observed in the tert-/- No Cre group a decreased abundance of Alpha-proteobacteria and Planctomycetes along with an enrichment in Gamma-proteobacteria, Bacteroidia and Fibrobacteria when compared to other groups (Figure 3D, Supplementary figure 3A). Interestingly, while Alphaproteobacteria are known to inhibit host cell death and promote proliferation ${ }^{33}$, Gammaproteobacteria expansion is associated with early age-dependent loss of intestinal barrier integrity in flies ${ }^{31}$. 
Figure 3

A. Alpha Diversity:

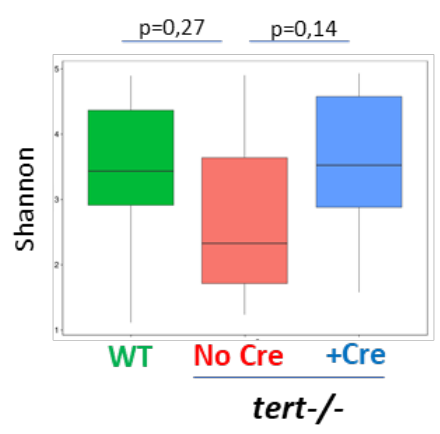

D.

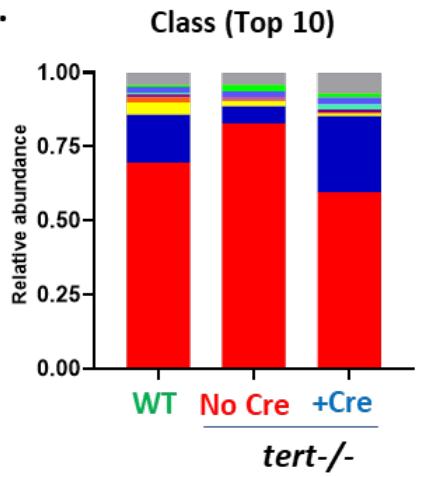

B. Beta Diversity:

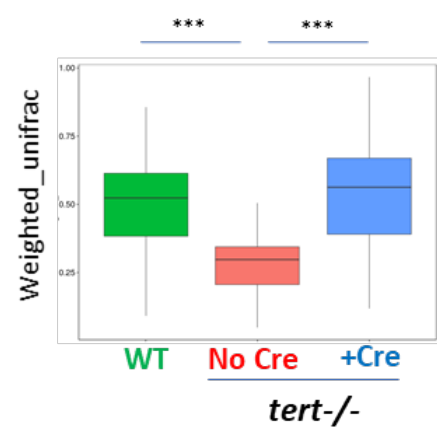

C. PCoA

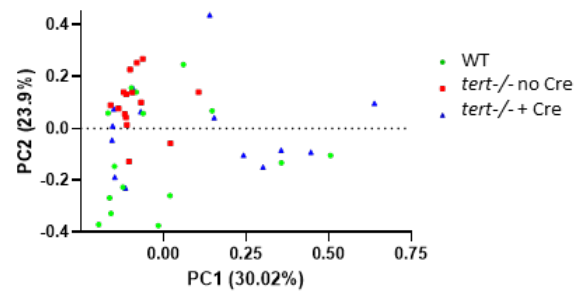

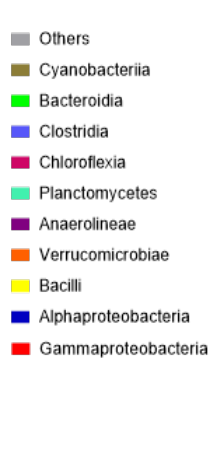

E.

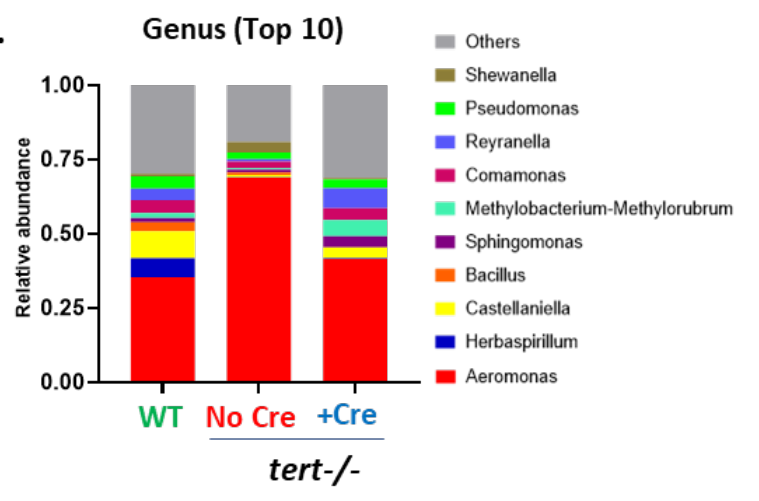

Figure 3: Gut specific telomerase activity rescues gut microbiota dysbiosis.

A. Quantification of microbiome alpha diversity (within samples) using Shannon index ( $N=14-15$; $p$-values were determine using Wilcoxon test) in the gut of 9-month-old fish. B. Quantification of microbiome beta diversity using weighed unifrac distance (within groups; $\mathrm{N}=14-15$; *** $\mathrm{p}<0.001$ using Tuckey test) in the gut of 9-monthold fish. C. Principal Coordinate Analysis (PCoA) of the beta diversity distance (weighted unifrac) in the gut of 9-month-old fish ( $\mathrm{N}=14-15)$. D. Relative abundance of top 10 bacteria classes in the microbiome of the 3 different groups in the gut of 9-month-old fish ( $\mathrm{N}=14-15)$. E. Relative abundance of top 10 bacteria genus in the microbiome of the 3 different groups in the gut of 9-month-old fish ( $\mathrm{N}=14-15)$. Telomere elongation in gut of tert-/- +Cre fish rescues gut microbiota composition and diversity to WT levels compared to tert-/- No Cre fish which exhibit gut microbiota dysbiosis.

Similarly, at the genus level, Alpha-proteobacteria Reyranella and Defluviimonas were reduced while Gamma-proteobacteria Aeromonas and Shewanella along with Bacteroides, a Bacteroidia-related genus, were enriched in tert-/- No Cre fish when compared to other groups (Figure 3E; Supplementary figure 3B). Both Shewanella and Aeromonas genus were described as deleterious in human, with Shewanella causing intra-abdominal infections ${ }^{34}$, and Aeromonas being associated with inflammatory bowel diseases and inflammation ${ }^{35,36}$. Interestingly, within the Aeromonas genus, A. veronii species were strikingly overrepresented in tert-/- No Cre compared to the other groups (Supplementary figure 3C). From the Bacteroidia class, $B$. uniformis, P. merdae and B. ovatus were similarly enriched in tert-/- No Cre and can be considered as "pathobionts" that profit from a dysregulated environment to overtake commensal symbionts and become pathogenic ${ }^{37-39}$. Overall, the analysis of gut microbiota composition revealed a dysbiotic microbiota in the tert-/- No Cre containing less diverse and more pathogenic bacterial community compared to WT that was reverted by gut-specific telomerase activity. 


\section{Local effects: Gut-specific telomerase activity recovers tissue metabolic profile}

Metabolomic analyses can determine the physiological and pathological status of a tissue by measuring metabolites representative of intrinsic and extrinsic factors. Changes in metabolism have been associated with aging and might reflect cellular defects, such as gradual mitochondrial dysfunction with age ${ }^{40,41}$. Similarly, we reported that, by 9 months of age, tert-/gut is affected by mitochondrial dysfunction accompanied by lower ATP and high ROS levels ${ }^{17}$. In order to gain insight on the metabolic profile of tert-/- No Cre and the extent of metabolic improvement by telomerase activity, we performed a metabolic analysis of whole intestinal extracts.

Clustering analyses on metabolomic profiles revealed that both WT and tert-/- +Cre samples clustered tightly while tert-/- No Cre samples differed from other groups (Figure 4AC). Notably, a significant number of metabolites were reduced (621) or enriched (141) in both WT and tert-/- +Cre when compared to tert-/- No Cre fish (Supplementary figure 4A). Consistent with our previous work ${ }^{17}$, we observed a drastic reduction of energetic metabolites in tert-/- No Cre such as ATP, ADP, NADH, NADPH and CoA compared to the other groups (Figure 4D). Following the anaerobic glycolysis pathway, we noticed lower levels of glucose6-phosphate and fructose-1,6-bisphosphate and higher amounts of pyruvate and lactate (Supplementary figure 4B). Considering that glucose did not vary greatly between the groups, our results suggest that the gut of tert-/- No Cre acquired higher levels of anaerobic glycolysis. We also detected higher pentose shunt activity in tert-/- No Cre gut, evidenced by increased amounts of ribose-5-phosphate and eythrose-4-phosphate (Supplementary figure 4C). Interestingly, except for citrate levels, all the detected metabolites of the citric acid cycle were elevated in the tert-/- No Cre fish compared to the other genotypes (Supplementary figure 5A). Altogether, the gut energetic metabolism of tert-/- No Cre fish appears to be engaged in uncoupled oxidative phosphorylation, consistent with the previously observed damaged mitochondria and higher production of ROS and that, by expressing tert transgene in the gut, the metabolic alterations is reverted in the entire tissue.

Among the detected amino acids, methionine was significantly enriched in tert-/- No Cre gut compared to the other genotypes (Figure 4E). We also observed an overall increase in methionine metabolites in the mutant gut that might be allowed by higher levels of nicotinamides.

In line with our previous results depicting higher inflammation of tert-/- No Cre fish, we observed an overall increase in the arachidonic metabolism with higher levels of proinflammatory molecules, such as prostaglandins and leukotrienes (Figure 4F). Consistently, we detected lower amounts of anti-inflammatory resolvin D2 in tert-/- No Cre fish when compared to the other groups. Interestingly, the steroid pathway was also enriched in tert-/- No Cre fish. Not only the stress hormone cortisol but also female hormones (such as 16-Oxoestrone or Estradiol) were elevated in male tert-/- No Cre fish (Figure 4G). Overall, our unbiased metabolomic analysis described an altered metabolism profile in tert-/- No Cre that was 


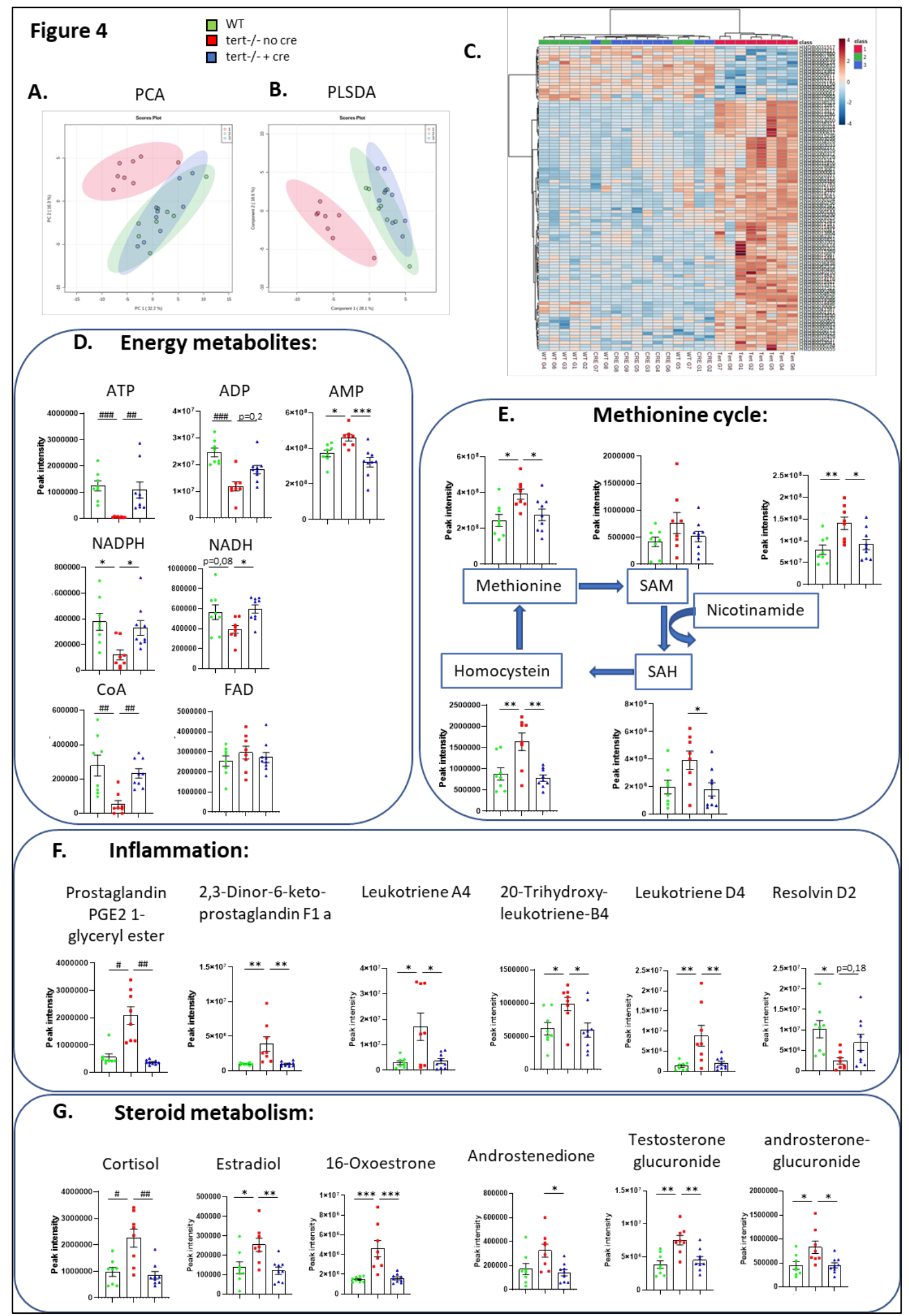


Figure 4: Gut-specific telomerase activity rescues gut metabolomic profile. clustering analysis based on untargeted metabolomic data of 9-month-old gut samples. A clustering between tert/ + +Cre and WT while tert-/- No Cre group was clearly distinguishable from the other ( $\mathrm{N}=8-9$ per group). The score plot is represented with a confidence ellipse of $95 \%$. D-G. Metabolomic analysis of energy metabolites (D.), methionine cycle pathway (E.), inflammatory metabolites (F.) and steroid metabolism (G.) in of 9-month-old gut samples. Metabolic alteration seen in gut of tert-/- No Cre fish are reverted to WT profile in tert-/- +Cre fish. All data are represented as mean $+/$ - SEM $(\mathrm{N}=8-9$ per condition; * $\mathrm{p}$-value $<0.05$; $* *$ p-value $<0.01$, *** $\mathrm{p}$-value $<0.001$, using one-way ANOVA and post-hoc Tuckey tests; \# p-value $<0.05$; \#\# p-value $<0.01$, \#\#\# p-value $<0.001$, using Kruskal-Wallis and post-hoc Dunn's tests).

\section{Systemic effects: Gut-specific telomerase expression rescues male fertility}

In light of the alterations of steroid metabolic profile observed in the gut of tert-/- No Cre and to explore the systemic impact of a gut-specific telomerase expression, we analysed aging phenotypes in the male reproductive system. As previously described ${ }^{14,17}$, we observed reduced cell proliferation and high senescence in testes of tert-/- No Cre fish (Figure 5A-D). Surprisingly, expression of the tert transgene specifically in the gut of tert-/- mutants led to a recovery of cell proliferation in the testes. Moreover, SA- $\beta$-Gal and p15/16 mRNA levels were reduced to WT levels in tert-/- +Cre testes, while p21 mRNA levels remained similar to tert-/No Cre fish. Similar to what we observed in the gut and consistent to our previous results for 9-month-old fish $^{17}$, apoptotic cell number were similar among the three genotypes (Supplementary figure 1B). Therefore, gut-specific telomerase activity rescues both proliferation and senescence in the reproductive system.

To ensure that these effects were not due to Fabp2 enterocyte promoter expression in other tissues, we performed RT-qPCR experiments on the testes of the three groups studied. While a clear induction of the tert transgene was observed in the gut of 9-month-old tert-/- +Cre fish compared to tert-/- No Cre fish, no expression of the transgene was detected in the testes (Supplementary figure 6A-B). Accordingly, we could not detect any difference on total tert mRNA levels when comparing testes of both groups. Consistently, there was no observable telomere elongation in the testes of tert-/- +Cre fish when compared to tert-/- No Cre (Supplementary figure 6C-E). As expected, in both these tert-/- groups, telomere length was similarly shorter when compared to WT fish. These control experiments support the systemic role of gut-specific telomerase activity in tert-/- fish.

Histopathology analysis of testes showed atrophy with a drastically reduced mature spermatids' content in the tert-/- No Cre fish compared to WT (Figure 5E-F), similar to what we previously reported ${ }^{14,17}$. In line with the cell proliferation and senescence rescue, gutspecific telomerase activity recovered these morphological defects. As in the gut, the increased neutrophil and eosinophil testes infiltrates present in tert-/- No Cre when compared to WT were also reverted in the tert-/- +Cre fish (Figure 5G-H).

Finally, male fertility decreases during natural aging of zebrafish and mice. This loss of fertility is accelerated in the murine and fish premature tert-/- aging models ${ }^{14,42}$. To provide further functional insight on the effect of gut aging delay on the reproductive function, we performed a male fertility assay where 9-month-old males of the three groups were individually crossed with young WT females. Percentage of eggs spawned by young females that were fertilized were scored as male fertility index. In accordance with a reduction of mature 
bioRxiv preprint doi: https://doi.org/10.1101/2022.01.10.475664; this version posted January 11, 2022. The copyright holder for this preprint (which was not certified by peer review) is the author/funder, who has granted bioRxiv a license to display the preprint in perpetuity. It is made available under aCC-BY-ND 4.0 International license.

spermatids' content, at 9 months of age, tert-/- No Cre male fish exhibit a drastic reduction of fertility compared to WT fish (Figure 5J). Strikingly, we observed a full recovery of male fertility in the tert-/- +Cre fish. Therefore, gut-specific telomerase activity not only improves cellular and morphological defects of the male reproductive system of tert-/- fish, but also rescues their age-dependent loss of fertility.

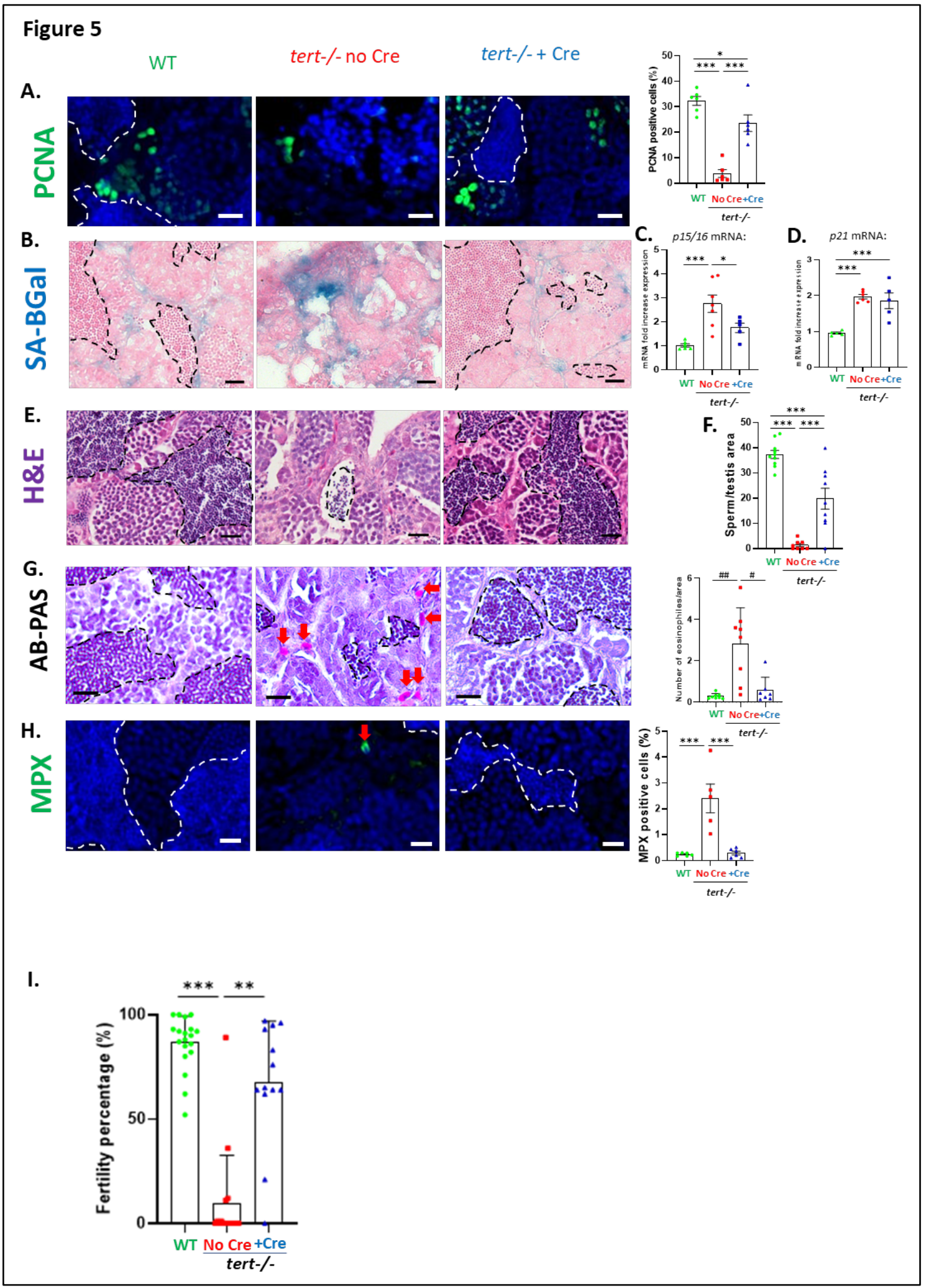


Figure 5: Gut-specific telomerase activity rescues testis aging phenotypes. quantification (right panel) in 9-month-old testis tissues. B. Representative image of SA- $\beta$-Gal staining of 9month-old testis cryosection. C-D. RT-qPCR analysis of senescence-associated genes $p 15 / 16(\mathbf{C}$.) and $p 21$ (D.) expression in 9-month-old testis samples. Delaying gut aging in tert-/- +Cre fish ameliorates testis proliferation and senescence compared to tert-/- No Cre fish. E. Representative hematoxylin and eosin-stained sections of testis from 9-month-old fish. F. Quantification of mature spermatids area over total testis area measured on histology images of 9-month-old fish testis. G. Representative AB-PAS staining images of 9-month-old fish testis (left panel). Number of pink-staining eosinophile cells (red arrows) are quantified in the right panel. H. Representative immunofluorescence images of neutrophil staining (MPX marker; left panel) and quantification (right panel) in 9month-old testis tissues. I. Quantification of male fertility of 9-month-old fish determined by counting the percentage of fertilized eggs (detected by successful embryogenesis events) after crossing individually 9-monthold males with a young (3-6 month) WT female. Tert mRNA expression in gut of tert-/- (tert-/- +Cre fish) have beneficial systemic effects by improving testis function and reducing testis inflammation compared to tert-/- No Cre fish. Scale bar: $20 \mu \mathrm{m}$. Dashed lines delineate mature spermatids area. All data are represented as mean +/SEM ( $\mathrm{N}=6-8$ per condition; * $\mathrm{p}$-value $<0.05 ; * * \mathrm{p}$-value $<0.01$, ${ }^{* * *} \mathrm{p}$-value $<0.001$, using one-way ANOVA and post-hoc Tuckey tests; \# p-value $<0.05$; \#\# p-value $<0.01$, \#\#\# p-value $<0.001$, using Kruskal-Wallis and post-hoc Dunn's tests). All RT-qPCR graphs are representing mean \pm SEM mRNA fold increase after normalization by rps 11 gene expression levels.

\section{Systemic effects: Gut-specific telomerase activity improves health and extends lifespan}

We then investigated whether the beneficial effects of gut aging delay could be observed beyond the reproductive system. Given the importance of anemia in telomeropathy patients ${ }^{43,44}$, we specifically investigated for improvements in the kidney marrow, the adult hematopoietic organ in zebrafish. Similar to testes and consistent with the increasing anemic profile, we detected lower levels of cell proliferation in the kidney marrow of tert-/- No Cre fish when compared to WT fish (Figure 6A). Notably, upon expression of tert transgene in the gut, proliferation rate in the kidney marrow was normalized to WT levels. Similarly, the increased senescence of tert-/- No Cre was also rescued in the tert-/- + Cre fish (Figure 6B-D). Like in the gut and testes, no differences in apoptosis were detected between the three groups (Supplementary figure 1C). We ruled out Fabp2-dependent expression of telomerase in the kidney marrow of tert-/- +Cre fish as we were unable to detect neither tert transgene expression nor differences in total tert mRNA expression when compared to tert-/- No Cre fish (Supplementary figure 6A-B). Consistently, a drastic shortening of telomere length was observed in tert-/- +Cre kidney marrow at 9 months of age similar to the telomere length of tert-/- No Cre fish (Supplementary figure 6E-H). Therefore, as in testes, gut-specific telomerase activity counteracted telomere-dependent cellular defects in the hematopoietic organ of tert-/fish.

In contrast to other analysed organs, we detected a depletion of immune cells such as eosinophils and neutrophils in the kidney marrow of tert-/- No Cre when compared to WT fish (Figure 6F-G). These numbers were reverted to WT levels in tert-/- +Cre fish. Our results suggest a decreased reserve pool of eosinophils and neutrophils in tert-/- No Cre that is rescued

402 by gut-specific telomerase activity. Decline of immune cells in the kidney marrow may constitute an early sign of hematopoietic dysfunction, comparable to the bone marrow failure described in telomeropathy patients ${ }^{43,44}$. 


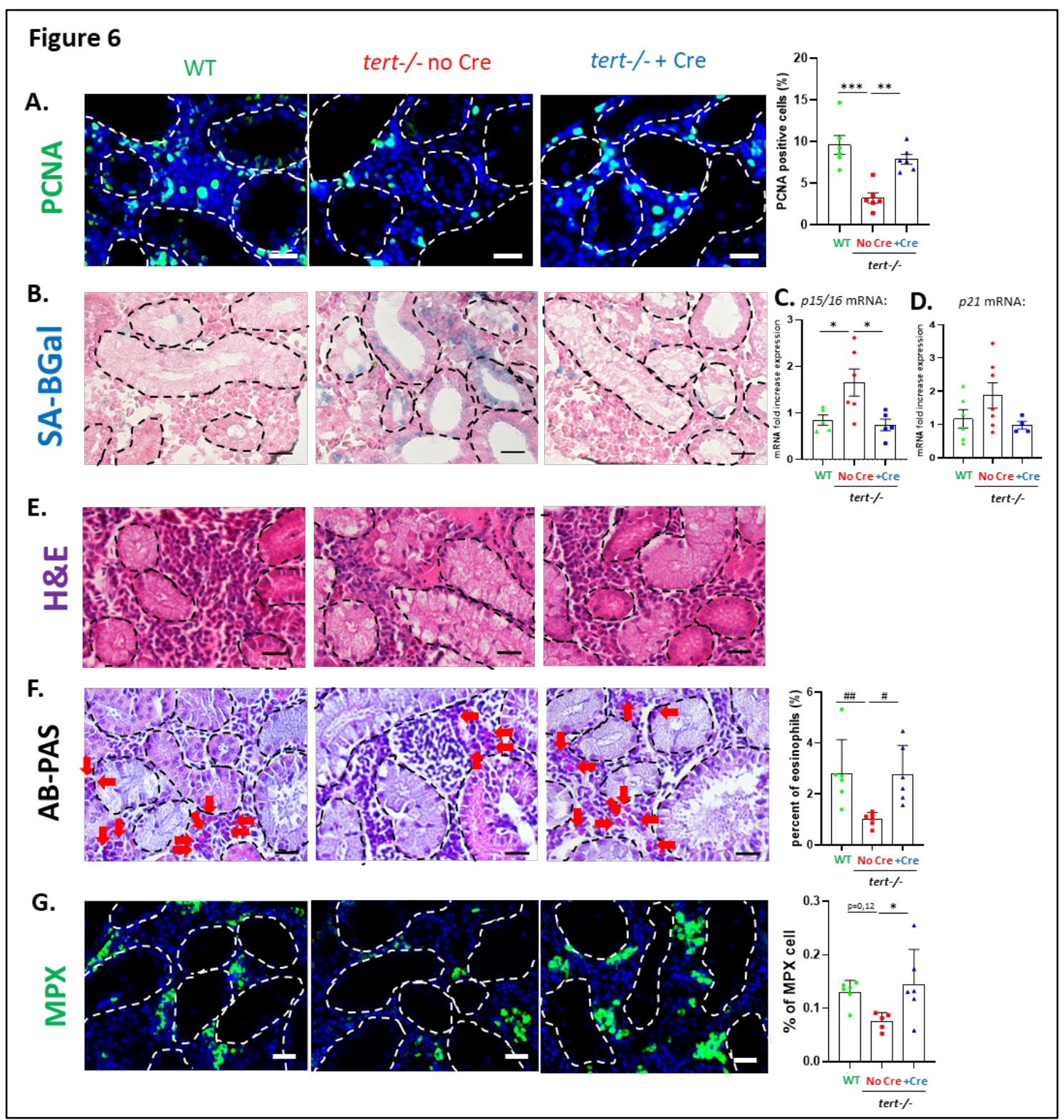

Figure 6: Gut-specific telomerase activity rescues aging of the hematopoietic system (kidney marrow).

A. Representative immunofluorescence images of proliferation staining (PCNA marker; left panel) and quantification (right panel) in 9-month-old kidney marrow (KM) tissues. B. Representative image of SA- $\beta$-Gal staining of 9-month-old KM cryosection. C-D. RT-qPCR analysis of senescence-associated genes p15/16 (C.) and p21 (D.) expression in 9-month-old KM samples. Delaying gut aging in tert-/- +Cre fish ameliorates KM proliferation and senescence compared to tert-/- No Cre fish. E. Representative hematoxylin and eosin-stained sections of KM from 9-month-old fish. F. Representative AB-PAS staining images of 9-month-old fish KM (left panel). Number of pink-staining eosinophile cells (red arrows) are quantified in the right panel. G. Representative immunofluorescence images of neutrophil staining (MPX marker; left panel) and quantification (right panel) in 9month-old KM tissues. Tert mRNA expression in gut of tert-/- (tert-/- +Cre fish) have beneficial systemic effects by improving neutrophil and eosinophil pool in compared to tert-/- No Cre fish. Scale bar: $20 \mu \mathrm{m}$. Dashed lines delineate kidney tubules. All data are represented as mean $+/$ SEM $(\mathrm{N}=5-8$ per condition; * $\mathrm{p}$-value $<0.05 ; * * \mathrm{p}-$ value $<0.01, * * *$ p-value $<0.001$, using one-way ANOVA and post-hoc Tuckey tests; \# p-value $<0.05$; \#\# pvalue $<0.01$, \#\#\# p-value $<0.001$, using Kruskal-Wallis and post-hoc Dunn's tests). All RT-qPCR graphs are representing mean \pm SEM mRNA fold increase after normalization by rps 11 gene expression levels. 
Finally, considering that delaying gut aging rescued the aging phenotypes of distant organs, such as testes and kidney, we wondered whether telomerase activity in the gut of tert/- would influence zebrafish lifespan. We grew male and female zebrafish of the three different groups and measured their life expectancy. As described previously ${ }^{14-16}$, accelerated telomere shortening of tert-/- No Cre fish reduces their lifespan to 12-18 months compared to $>42$ months in WT fish (Figure 7). Strikingly, delaying gut aging was sufficient to significantly extend the average lifespan of tert-/- fish. Average lifespan of tert-/- No Cre recovered from 17 months to 24 months in tert-/- +Cre fish. However, this was not sufficient to fully rescue life expectancy to WT levels, suggesting that telomere shortening in other organs may become limiting in later stages. Therefore, counteracting gut aging not only delays aging of distant organs, but it is sufficient to extend lifespan of tert-/- mutants by $40 \%$.

Figure 7

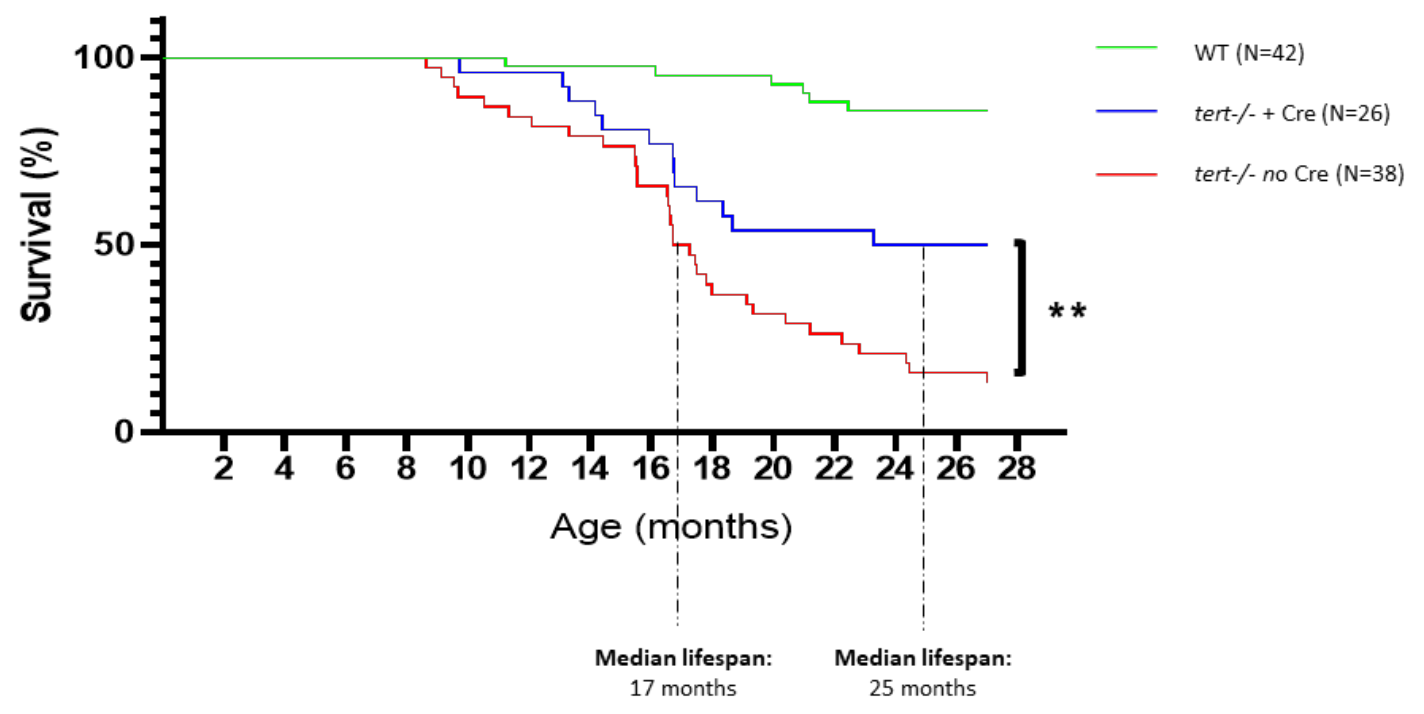

Figure 7: Gut-specific telomerase activity extends lifespan of tert-/- zebrafish. (** p-value $<0.01$ using Log-rank test). 


\section{DISCUSSION:}

The gut is a central organ in aging and it constitutes one of the most extensive and selective living barriers to the external environment. Besides its nutrient uptake function, it plays an important role in immune modulation and support a complex interaction with gut microbiota 4 .

In our study, we show that enterocyte-specific telomerase activity in tert-/- fish is sufficient to prolong maintenance of gut homeostasis with age. Not only it rescues proliferative defects and cell senescence, but also tissue integrity while reducing tissue inflammation. Interestingly, rescue of gut aging was observed even with a mild but significant telomere extension. Broad telomerase expression counteracts degenerative phenotypes of late generation tert-/- mice ${ }^{45,46}$. Improvement of aging phenotypes was observed not only in the gut but also in other organs such testes, spleen, brain or skin. However, in these studies, tert expression was not targeted to a specific organ, such as the gut. Consistent with our observations, the dePinho lab recently showed that telomere shortening in the mouse gut activates inflammation by a mechanism involving $\mathrm{YAP}^{47}$. In this work, a mosaic expression of tert in LGR5 positive cells of tert-/- mice partially ameliorated intestinal function and reduced inflammation of this tissue, but no systemic effects were reported apart from body weight rescue and a modest increase in survival. Consistently, we show that YAP target genes were likewise induced in tert-/- No Cre fish. These were rescued in the tert-/- + Cre fish that, not only reverted the YAP pathway, but also rescued local inflammation. Moreover, we now show that counteracting gut telomere dysfunction also delays remote organ dysfunction and overall organismal aging. It is worth noting that trace amounts of $f a b p 2$ transcripts were previously reported in zebrafish in the liver, brain and kidney marrow but not in testis ${ }^{48}$. While in our study, we did not detect any fabp 2 promoter-dependant transgene expression and telomere extension in kidney marrow, we cannot exclude that a negligible tert transgene expression in non-proliferative tissues such as brain and liver might participate to the systemic improvement.

We report that delaying telomere-dependent gut aging has beneficial systemic effects. Expression of tert transgene exclusively in the gut of tert-/- fish reverted cellular defects in the reproductive (testes) and the hematopoietic (kidney marrow) systems, namely reduced cell proliferation and senescence. At the organ level, improving cellular turnover and reducing inflammation in testes allowed for replenishment of mature spermatids leading to functional rescue of male fertility. In parallel, we observed that neutrophil and eosinophil pools were restored in the hematopoietic system. Strikingly, in line with a systemic recovery, counteracting intestinal aging of tert-/- zebrafish extended lifespan by $40 \%$. Notably, our study indicates that proliferative organs such as the reproductive or hematopoietic systems can conserve regenerative capacity even in a context of short telomeres. This has been observed in the rescue of telomerase deficiency by tp53 mutations in several organisms, namely mice and zebrafish $^{15,49}$. Thus, our study anticipates that maintenance of proliferative capacity and tissue integrity relies on external signals induced by an aging gut.

How would gut aging influence the entire organism? The recent years have seen a flurry of studies supporting the role of inflammation/SASP in inducing paracrine senescence in remote tissues ${ }^{50,51}$. Senescent cells accumulate with age in tissues and promote aging by secreting molecules such as inflammatory cytokines, chemokines and other molecules, also known as $\mathrm{SASP}^{51}$. Remarkably, clearance of these cells delay age-associated defects and lead 
to lifespan extension ${ }^{5,6}$. We previously reported that some organs, such as kidney marrow, exhibit onset of cellular senescence before reaching critically short telomeres during zebrafish aging $^{14,15}$. Interestingly, in the present study, we observed that enterocyte-specific telomerase activity in tert-/- fish not only counteracted senescence in the gut but also in distant organs. A paracrine signaling driven by inflammation/SASP factors secreted by an aged gut with short telomeres might therefore promote senescence in remote organs in tert-/- No Cre zebrafish. This mechanism would affect cell proliferation systemically and eventually lead to loss of tissue homeostasis in the entire organism.

Over the last decades, gut microbiota has captured the scientific community's interest by its implications in the etiology of several diseases including inflammatory bowel disease, type 2 diabetes, hypertension, liver diseases and depression ${ }^{52}$. Modification of gut microbiota dysbiosis has been linked to aging ${ }^{23,24}$ and is involved in age-related systemic inflammation ${ }^{25}$. We show that tert-/- No Cre fish are afflicted by gut microbiota dysbiosis, with reduced population diversity and enrichment of pathogenic bacteria. Gut-specific telomerase activity recapitulated the bacterial diversity and composition observed in WT fish. Therefore, delaying gut aging counteracted gut microbiota dysbiosis. We anticipate that, as gut telomere shortening becomes limiting, increasingly dysbiotic microbiota exacerbates the age-dependent effects over the entire organism through its microbial components and/or by inducing systemic inflammation. This idea is supported by a recent work showing that stool transfers from young to middle-aged individuals was sufficient to extend lifespan of short-lived killifish ${ }^{53}$.

By analysing the metabolic profile of the gut in our model, we noticed an overall accumulation of methionine and its metabolites (SAM, SAH and homocystein) that is reverted to WT levels in tert-/ + Cre fish. Similar enrichment of methionine and its metabolites with age were reported in human and mice ${ }^{40,54}$. Interestingly, dietary methionine restriction or impeding SAM accumulation extends lifespan in different animal models ${ }^{4,55,56}$. Moreover, hyperhomocysteinemia has been implicated in several age-related disorder such as cardiovascular diseases, osteoporosis, renal and cognitive dysfunctions ${ }^{54}$. Mechanistically, deleterious effects of methionine and its metabolites involves DNA methylation drift, mTOR activation, inflammation and oxidative stress ${ }^{4,41,56}$. While it remains unclear why the levels of methionine and its metabolites are enriched in tert-/- gut, we anticipate/propose that propagation of these molecules throughout the zebrafish organism may precipitate systemic aging.

A growing list of evidence described the implication of the gut in different systemic physiological and pathological processes, often involving gut microbiota. Overall, the present work describes a central role of telomere shortening in the gut during the aging of a vertebrate organism. While it provides several mechanistic clues, it remains unclear how this organ influences aging of the entire organism. This includes microbiota dysbiosis, inflammation/SASP and dysregulation of methionine metabolism. Finally, our study demonstrates that targeting aging of a unique organ, the gut, is an exciting strategy to extend health span and lifespan. 
MATERIAL AND METHODS:

\section{Ethics statement}

Zebrafish work was conducted according to local and international institutional guidelines and were approved in France by the Animal Care Committee of the IRCAN, the regional (CIEPAL Cote d'Azur \#697) and national (French Ministry of Research \#27673-2020092817202619) authorities and in Portugal by the Ethical Committee of the Instituto Gulbenkian de Ciência and approved by the competent Portuguese authority (Direcção Geral de Alimentação e Veterinária; approval number: 0421/000/000/2015).

\section{Plasmid construct}

Zebrafish tert $c D N A$ was obtained using TertFL- pCR-II-Topo plasmid kindly provided by S. Kishi laboratory ${ }^{57}$. Using Gibson assembly recombination methods, tert cDNA and $e C F P$ cDNA were linked by $T 2 A$ sequence and inserted into Ubi: loxP-dsRed-loxP-EGFP vector plasmid (a kind gift from Zon's lab derived from ubi:Switch and lmo2: Switch contructs $^{58}$ ). Then, the intestine- specific intestinal fatty acid binding protein promoter $(-2.3 \mathrm{~kb} f a b p 2$-also called ifabp-) was amplified by high fidelity PCR (iProof ${ }^{\mathrm{TM}}$ High-Fidelity DNA Polymerase; Bio-Rad, Hercules, CA, USA) from p5E-2.3ifabp plasmid (kindly gifted by J. Rawls laboratory). $-2.3 \mathrm{~kb}$ fabp2 PCR product was then cloned into the Ubi: LoxPdsRed-loxP-tert-T2A-CFP using sfI/ FseI digestion to provide the final construct: Fabp2: LoxP-dsRedloxP-tert-T2A-CFP.

\section{Generation of transgenic fish}

Tol2 mRNA was synthesized with SP6 RNA polymerase from pCS2FA-transposase plasmid (Tol2Kit) using mMESSAGE mMACHINE SP6 transcription kit (Invitrogen; Cergy Pontoise, France). One-cell stage zebrafish embryos were micro-injected with $1,4 \mathrm{~nL}$ of a mixture containing $25 \mathrm{ng} / \mu \mathrm{L}$ of linearized plasmid and $100 \mathrm{ng} / \mu \mathrm{L}$ of Tol $2 \mathrm{mRNA}$, diluted with RNase-free water. Injected fish were raised to adulthood and germline transmitting fish were then selected and out-crossed to wild type $\mathrm{AB}$ until obtaining a single copy transgenic line $\mathrm{Tg}$ (Fabp2: LoxP-dsRed-loxP-tert-T2A-CFP).

\section{Zebrafish lines and maintenance}

Zebrafish were maintained in accordance with Institutional and National animal care protocols. Generation and maintenance of the telomerase mutant line tert $\mathrm{AB} / \mathrm{hu} 3430$ (referred in this work as tert+/-) were previously described ${ }^{14,15,17}$. This line was outcrossed with $\operatorname{Tg}$ (Fabp2: LoxP-dsRed-loxPtert-T2A-CFP) line to obtain a stock line combining both transgenics. All stocks were kept in heterozygous form for tert mutation and maintained strictly by outcrossing to $A B$ strains to avoid haploinsufficiency effects in the progeny.

Experimental fish were obtained by crossing tert+/- fish with tert+/-; Fabp2: LoxP-dsRed-loxP-tert$T 2 A-C F P$. The generated sibling embryos were then micro-injected with $1.4 \mathrm{~nL}$ of either $25 \mathrm{ng} / \mu \mathrm{L}$ Cre mRNA diluted in RNase-free water (Cre induced fish), or RNase-free water alone (mock injected fish). This experimental set up provided sibling fish that are either tert-/-; Fabp2: LoxP-dsRed-loxP-tert-T2A$C F P$ (mock injected tert-/- referred to as "tert-/- No Cre"), tert-/-; Fabp2: tert-T2A-CFP (Cre induced tert-/- referred to as "tert-/- +Cre") or tert+/+; Fabp2: LoxP-dsRed-loxP-tert-T2A-CFP (mock injected wild type referred to as "WT"). Overall characterization of these three genotypes was performed in F1 sibling animals at 9 months of age. Due to a male sex bias in our crosses, that affected mostly tert-/progeny, we were unable to obtain significant numbers of females for analysis and so all of our data except survival analysis are restricted to males.

\section{Senescence-associated beta-galactosidase staining}

Tissues were fixed with $4 \%$ PFA during 3 hours at $4^{\circ} \mathrm{C}$. After being washed in PBS, they were incubated in $30 \%$ sucrose (Sigma, MO, USA) at $4^{\circ} \mathrm{C}$ until sinking (24-48hours). Fixed tissues were then embedded in OCT medium (M-M France, Brignais, France) and kept at $-80 \mathrm{C}$. Senescence-associated betagalactosidase staining was then performed on slides of $5 \mu \mathrm{m}$ cryosections using Senescence BetaGalatosidase staining kit (\#9860, Cell Signalling Technology, Danvers, MA, USA) following manufacturer's instructions. After 16h (testis, kidney marrow) or 3h (gut) incubations with the X-Gal 
staining solution at $37^{\circ} \mathrm{C}$, slides were washed with PBS and counterstained for one minute with Nuclear Fast Red (NFR) solution (Sigma, MO, USA) prior to being dehydrated and mounted.

\section{Telomere restriction fragment (TRF) analysis by Southern blot}

Isolated tissues were first lysed at $50^{\circ} \mathrm{C}$ overnight in lysis buffer (Fermentas \#K0512; Waltham, MA, USA) supplemented with $1 \mathrm{mg} / \mathrm{ml}$ Proteinase K (Sigma, MO, USA) and RNase A (1:100 dilution, Sigma, MO, USA). Genomic DNA was then extracted by equilibrated phenol-chloroform (Sigma, MO, USA) and chloroform-isoamyl alcohol extraction (Sigma, MO, USA). Same amounts of gDNA were digested with RSAI and HINFI enzymes (NEB, MA, USA) for $12 \mathrm{~h}$ at $37^{\circ} \mathrm{C}$. After digestion, samples were loaded on a $0.6 \%$ agarose gel, in $0.5 \%$ TBE buffer, and run on a CHEF-DRII pulse field electrophoresis apparatus (Bio-Rad). The electrophoresis conditions were as follow: initial swtich 1s, final switch $6 \mathrm{~s}$; voltage $4 \mathrm{~V} / \mathrm{cm}$; at $4^{\circ} \mathrm{C}$ for $20 \mathrm{~h}$. Gels were then processed for Southern blotting using a $1.6 \mathrm{~kb}$ telomere probe, (TTAGGG)n, labelled with [ $\alpha-32 \mathrm{P}]$-dCTP.

\section{Fertility assays}

In order to assess male fertility, 9-month-old male individuals from the three different genotypes were separately housed overnight in external breeding tanks with a single young (3-6 month old) WT female. Breeding pairs were left to cross and to lay eggs the following morning. Embryos were collected approximately 2 hours post fertilization (hpf) and allowed to develop at $28^{\circ} \mathrm{C}$. Assessment of egg fertilization and embryo viability was conducted between 2 and 4 hpf. At least 14 independent crosses were conducted for each genotype to evaluate male fertility. Only successful breeding trials, defined as events where clutch of eggs was laid by a female, were scored.

\section{Histology}

Zebrafish were sacrificed by lethal dose of $1 \mathrm{~g} / \mathrm{L}$ of MS-222 (Sigma, MO, USA), fixed for $72 \mathrm{hr}$ in 10\% neutral buffered formalin and decalcified in $0.5 \mathrm{M}$ EDTA for $48 \mathrm{hr}$ at room temperature. Whole fish were then paraffin-embedded in order to perform five micrometer sagittal section slides. Slides were stained with haematoxylin and eosin for histopathological analysis. In parallel, slides were stained by Alcian Blue (AB) solution pH 2.5 (Sigma, MO, USA) followed by Periodic acid-Schiff staining (kit \#395B, Sigma, MO, USA) according to manufacturer's instructions. Microphotographs ( $\mathrm{N}>=6$ fish per genotype) were acquired in a Leica DM4000B microscope coupled to a Leica DFC425 C camera.

\section{Immunofluorescence}

Deparaffinized and rehydrated slides were microwaved $20 \mathrm{~min}$ at $550 \mathrm{~W}$ in citrate buffer ( $10 \mathrm{mM}$ Sodium Citrate, $\mathrm{pH}$ 6) to allow for antigen retrieval. Slides were washed two times in PBS for 5 minutes each and blocked for 1 hour at RT in 0.5\% Triton, 5\% normal goat serum in PBS (blocking solution). Subsequently, slides were incubated overnight at $4{ }^{\circ} \mathrm{C}$ with 1:50 dilution of primary antibody in blocking solution. The following primary antibodies were used: mouse monoclonal antibody against Proliferation Cell Nuclear Antigen (PCNA, sc56 Santa Cruz, CA, USA, 1:50 dilution) and rabbit polyclonal against myeloperoxidase (MPX, GTX128379; Irvine, CA, USA, 1:50 dilution). After two PBS washes, overnight incubation at $4^{\circ} \mathrm{C}$ was performed with 1:500 dilution of goat anti-rabbit or anti-mouse secondary antibodies Alexa Fluor 488 (Invitrogen; Cergy Pontoise, France). Finally, after DAPI staining (Sigma, MO, USA), slides were mounted DAKO Fluorescence Mounting Medium (Sigma, MO, USA). Apoptosis was detected using the In SituCell Death Detection Kit (Roche, Bâle, Switzerland) as previously described ${ }^{14,17}$. Briefly, deparaffinated sections were permeabilized by one hour incubation at $37^{\circ} \mathrm{C}$ with $40 \mu \mathrm{g} / \mathrm{ml}$ Proteinase $\mathrm{K}$ in $10 \mathrm{mM}$ Tris- $\mathrm{HCl} \mathrm{pH} \mathrm{7.4.} \mathrm{After} \mathrm{being} \mathrm{washed} \mathrm{with}$ PBS, slides were incubated one hour at $37^{\circ} \mathrm{C}$ with TUNEL labelling mix (according to manufacturer's instructions) prior to DAPI staining and mounting.

Immunofluorescence images were acquired on Delta Vision Elite (GE Healthcare, Chicago, IL, USA) using a OLYMPUS 20x/0,75 objective. For quantitative and comparative imaging, equivalent image acquisition parameters were used. The percentage of positive nuclei was determined by counting a total of $500-1000$ cells per slide ( $\mathrm{N}>=6$ zebrafish per genotype). 


\section{Real-time quantitative PCR and RNA sequencing}

634 Zebrafish were sacrificed by lethal dose of 1g/L of MS-222 (Sigma, MO, USA) and each tissue (gonads, gut and kidney marrow) were dissected and immediately snap-frozen in liquid nitrogen. RNA extraction was performed by disrupting individual tissues with a pestle in TRIzol (Invitrogen, UK) followed by chloroform extractions. Quality of RNA samples was assessed through BioAnalyzer (Agilent 2100, CA, USA). Retro-transcription into cDNA was performed using QuantiTect Reverse Transcription kit (Qiagen, Hilden, Germany).

Quantitative PCR (qPCR) was performed using FastStart Universal SYBR Green Master mix (Roche, Bâle, Switzerland) and an 7900HT Fast Real-Time PCR Detection System (Thermofisher, Waltham, MA, USA). qPCRs were carried out in triplicate for each cDNA sample. Relative mRNA expression was normalized against rps 11 mRNA expression using the $2^{-\Delta \Delta \mathrm{CT}}$ method as compared to controle condition. Primer sequences are listed in Supplementary table.

RNA sequencing was performed by the Beijing Genomics Institute (BGI; Hongkong), using for each condition, biological triplicates consisting for each of a pool of two individual tissues. DNAse treated total RNA samples were enriched for mRNAs using oligo dT magnetic beads. In turn, mRNAs were fragmented into $200 \mathrm{bp}$-size fragments and the first strands of cDNAs were synthesized by using random-hexamers. In order to generate library products, double stranded cDNAs from the second strand synthesis were then purified by magnetic beads followed by A-tailing and RNA adaptors ligation. The library was amplified with phi29 to make DNA nanoball (DNB) which had more than 300 copies of one molecular. Pair ended 150 bases reads were sequenced in the way of combinatorial Probe-Anchor Synthesis (cPAS) on DNBseq plateform and 100M clean reads per sample was generated. Raw data with adapter sequences or low-quality sequences was filtered SOAPnuke software developed by BGI. The RNA-seq reads were analyzed via an internal pipeline for transcript quantification, normalization, and comparison. Briefly, the human reference genome assembly vGRCh38 (retrieved from http://www.ensembl.org) and gencode annotation v37 (retrieved from https://www.gencodegenes.org/) were processed by gffread v0.12.2 to extract human reference transcriptome. Based on this extracted reference transcriptome, Salmon v1.4 was used to perform transcript quantification via quasi-mapping. RUVseq v1.20.0 was used for data transformation by "rlog" and data normalization by replicates. DESeq2 v1.26.0 was used for differentially expressed gene (DEG) analysis. The false discovery rate (FDR) cutoffs of 0.1 were explored for the DEG analysis. Based on the resulting DEG candidate gene lists, clusterProfiler v4.0 was employed for Gene Ontology (GO) analysis and Gene Set Enrichment Analysis (GSEA), based on which KEGG pathway enrichment analyses were further performed.

\section{Supplementary Table - List of primers used in}

RT-qPCR expression analysis.

\begin{tabular}{|l|l|}
\hline Gene name & Primer sequences \\
\hline$c d k n 2 a / b(\mathrm{p} 15 / 16)$ & $\begin{array}{l}\text { forward - 5' GAGGATGAACTGACCACAGCA 3' } \\
\text { reverse - 5' CAAGAGCCAAAGGTGCGTTAC 3' }\end{array}$ \\
\hline cdkn1a (p21) & $\begin{array}{l}\text { forward - 5' CAGCGGGTTTACAGTTTCAGC 3' } \\
\text { reverse - 5' TGAACGTAGGATCCGCTTGT 3' }\end{array}$ \\
\hline tnfa & $\begin{array}{l}\text { forward - 5' AGGCAATTTCACTTCCAAGGC 3' } \\
\text { reverse - 5' GGTCCTGGTCATCTCTCCAGT 3' }\end{array}$ \\
\hline tert & $\begin{array}{l}\text { forward - 5' CGGTATGACGGCCTATCACT 3' } \\
\text { reverse - 5' TAAACGGCCTCCACAGAGTT 3' }\end{array}$ \\
\hline tert transgene & $\begin{array}{l}\text { forward - 5' GCATGTTAGAAGACTTCCTCTGC 3' } \\
\text { reverse - 5' TTCCTCTCCCAGAATCCCCC 3' }\end{array}$ \\
\hline rps11 & $\begin{array}{l}\text { forward - 5' ACAGAAATGCCCCTTCACTG 3' } \\
\text { reverse - 5' GCCTCTTCTCAAAACGGTTG 3' }\end{array}$ \\
\hline il-6 & $\begin{array}{l}\text { forward - 5' TCAACTTCTCCAGCGTGATG 3' } \\
\text { reverse - 5' TCTTTCCCTCTTTTCCTCCTG 3' }\end{array}$ \\
\hline cyr 61 & $\begin{array}{l}\text { forward - 5' CCGTGTCCACATGTACATGGG 3' } \\
\text { reverse - 5' GGTGCATGAAAGAAGCTCGTC 3' }\end{array}$ \\
\hline ctgf & $\begin{array}{l}\text { forward - 5' ACTCCCCTCGTCAAAACACC 3' } \\
\text { reverse - 5' GGGACCGTATGTCTCCTCCT 3' }\end{array}$ \\
\hline flaudin-2 & $\begin{array}{l}\text { forward - 5' GCAACACCTCACTGCTGAAC 3' } \\
\text { reverse - 5' TTGCCCAGTAGGGGAGAAGA 3' }\end{array}$ \\
\hline
\end{tabular}




\section{Metagenomics}

gDNA was first extracted from isolated gut of sibling fish as described for Telomere restriction fragment (TRF) analysis. The V3-V4 hypervariable regions of bacterial 16S rRNA genes were amplified by PCR with Phusion ${ }^{\circledR}$ High-Fidelity PCR MasterMix (New England Biolabs, Ipswich, MA, USA) using specific primer as previously described ${ }^{59}$. PCR products were mixed at equal density ratios and purified with Qiagen Gel Extraction Kit (Qiagen, Germany). The sequencing libraries were generated using NEBNext ${ }^{\circledR}$ UltraTM DNA Library Prep Kit and sequenced on Illumina NovaSeq 6000 paired-end platform to generate 250 bp paired-end raw reads. Sequences analysis were performed using Uparse software with all the effective tags. Sequences with $\geq 97 \%$ similarity were assigned to the same OTUs. Representative sequence for each OTU was screened for further annotation. For each representative sequence, Mothur software was performed against the SSUrRNA database of SILVA Database for species annotation at each taxonomic rank (Threshold:0.8 1). QIIME and R were used to calculate alpha and beta diversity metrics and generate plots. Principal Coordinate Analysis (PCoA) was performed to get principal coordinates and visualize from complex, multidimensional data.

\section{Metabolomic analysis}

Each frozen gut sample was homogenized in methanol $600 \mu \mathrm{L}$ of methanol (HPLC grade, Merck Millipore, USA) and incubated overnight at $-20^{\circ} \mathrm{C}$. Tubes were vortexed and incubated overnight at $20^{\circ} \mathrm{C}$ for protein precipitation. After centrifugations, supernatants were removed, dried using a SpeedVAC concentrator (SVC100H, SAVANT, Thermo Fisher Scientific, Illkirch, France), resuspended in $80 \mu \mathrm{L}$ of a 20:80 acetonitrile-H2O mixture (HPLC grade, Merck Millipore) and stored at $-20^{\circ} \mathrm{C}$ until use for metabolomic analysis.

Chromatographic analysis was performed with the DIONEX Ultimate 3000 HPLC system coupled to a chromatographic column (Phenomenex Synergi $4 \mathrm{u}$ Hydro-RP 80A 250_3.0 mm) set at $40^{\circ} \mathrm{C}$ and a flow rate of $0.9 \mathrm{~mL} / \mathrm{min}$. Gradients of mobile phases (mobile phase A: $0.1 \%$ formic acid in water and mobile phase B: $0.1 \%$ formic acid in acetonitrile) were performed over a total of $25 \mathrm{~min}$. MS analysis was carried out on a Thermo Scientific Exactive Plus Benchtop Orbitrap mass spectrometer. The heated electrospray ionization source (HESI II) was used in positive and negative ion modes. The instrument was operated in full scan mode from $\mathrm{m} / \mathrm{z} 67$ to $\mathrm{m} / \mathrm{z} 1000$. The post-treatment of data was performed using the MZmine2 version 2.39 (http://mzmine.github.io/). Metabolites were identified using the Human Metabolome Database version 5.0 (http://www.hmdb.ca). We only used ions identified as $[\mathrm{M}+\mathrm{H}]^{+}$adducts in the positive mode and $[\mathrm{M}-\mathrm{H}]^{-}$adducts in the negative mode and ions found in all the samples after gap filling.

\section{Statistical analysis}

Graphs and statistical analyses were performed in GraphPad Prism8 software (San Diego, CA, USA), using one-way ANOVA test with Tuckey's post-correction or Kruskal-Wallis test with Dunn's post-hoc test. A critical value for significance of $\mathrm{p}<0.05$ was used throughout the study. For survival analysis, Log-rank tests were performed using GraphPad Prism8 in order to determine statistical differences of survival curves.

Untargeted metabolomic analysis of gut samples were processed using statistical analysis [one factor] modules proposed by MetaboAnalyst 5.0 (https://www.metaboanalyst.ca). For each comparison, peak intensities were Log transformed. Clustering analysis were performed using Principal Component Analysis (PCA), Partial Least Squares - Discriminant Analysis (PLS-DA), and Heatmap tools provided by MetaboAnalyst.

\section{ACKNOWLEDGEMENTS:}

We thank members from the Telomeres and Genome Stability and the Telomere Shortening and Cancer Laboratories for fruitful discussions. We are grateful to Leonor Saúde (Instituto de Medicina Molecular) and Ana Rita Araújo (IPMC) for critically reading our paper. This work was supported by the Fondation Arc pour la Recherche sur le Cancer (PJA20161205137) and the Fondation pour la Recherche Médicale 
FRM (EQU201903007804). MEM was supported by a postdoctoral fellowship from the Ville de Nice. This work was also supported by the Université Côte d'Azur - Académie 4 (Installation Grant: Action 2 - 2019) and the Howard Hughes Medical Institute International Early Career Scientist grant awarded to MGF. We thank the Instituto Gulbenkian de Ciência (IGC) histology unit, the IGC imaging unit, for assistance with experimental planning, sample processing and data collection and the IGC Fish Facility for excellent animal care. IGC Fish Facility is financed by Congento LISBOA-01-0145-FEDER022170, co-financed by FCT (Portugal) and Lisboa2020, under the PORTUGAL2020 agreement (European Regional Development Fund). The work was also performed using the PEMAV fish facility, Imaging core facility (PICMI) and the Genomics facilities at the IRCAN supported by FEDER, Région Provence Alpes-Côte d'Azur, Conseil Départemental 06, ITMO Cancer Aviesan (plan cancer), Cancéropole Provence Alpes-Côte d'Azur, Gis Ibisa, CNRS and Inserm.

\section{AUTHOR CONTRIBUTIONS}

T.P. and G.J-M. contributed to metabolomic analyses; J-X.Y. and D.K performed transcriptomics analyses; M.E.M. performed the experiments and carried out data analyses; M.E.M. and M.G.F. conceived the study, designed the experiments, and wrote the manuscript. M.G.F. supervised the work. 


\section{Supplementary figure 1}

A.
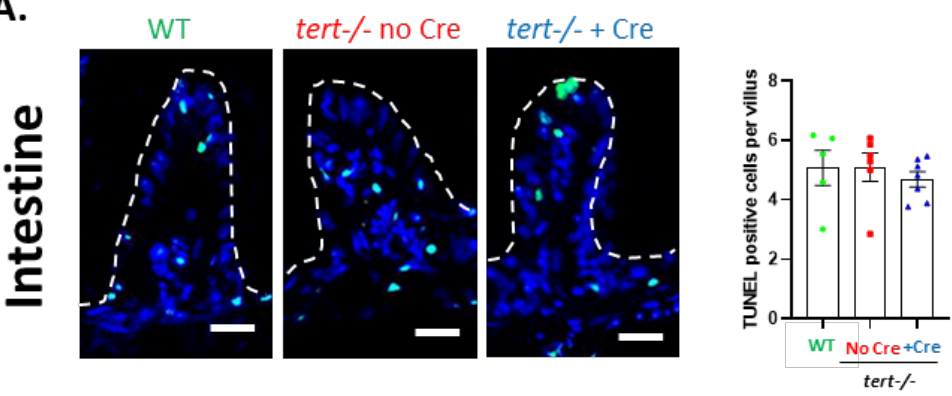

B.
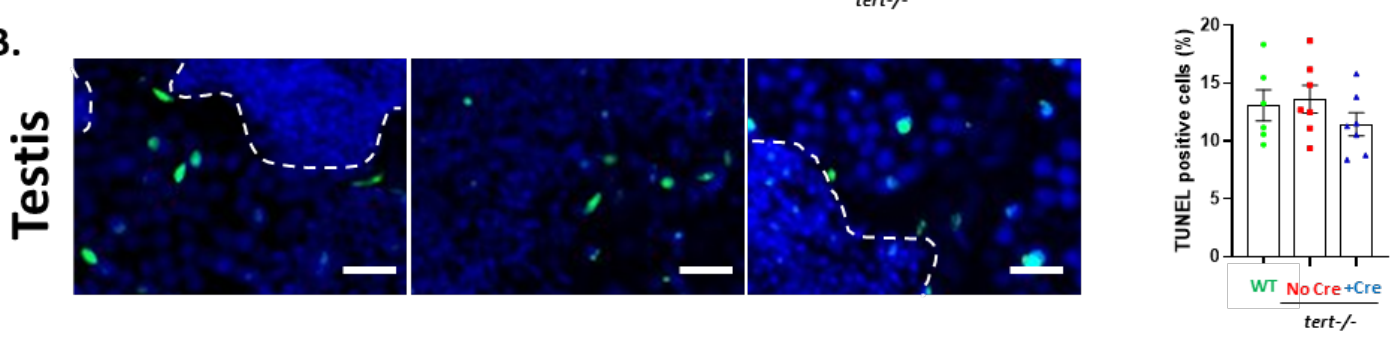

C.
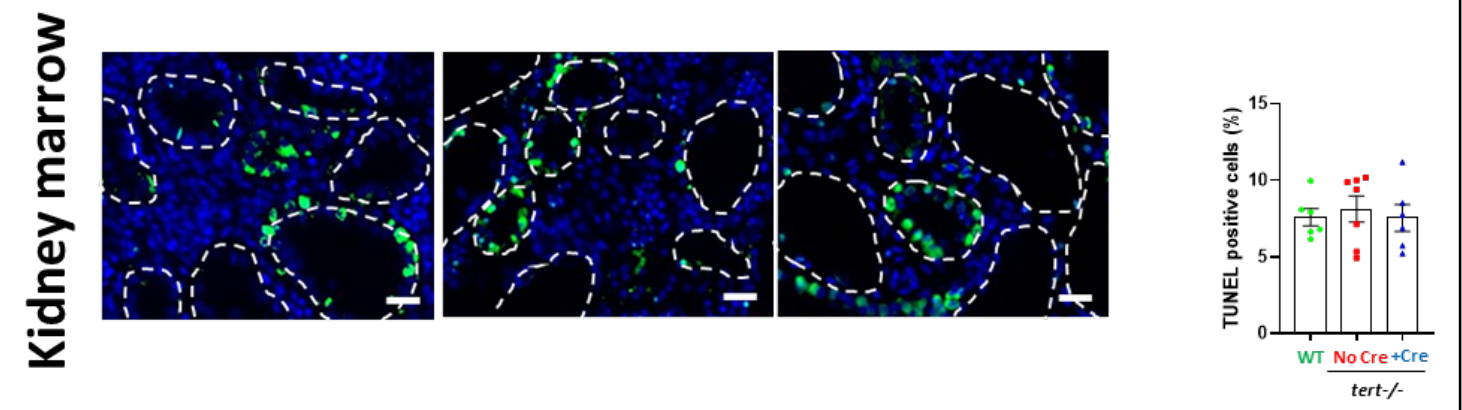

Supplementary figure 1: 9-month-old fish did not exhibit apoptosis differences between conditions.

A-C. Representative immunofluorescence images of apoptotic cell staining (TUNEL assay; left panel) and quantification (right panel) in gut (A.), testis (B.), or KM (C.) tissues of 9-month-old zebrafish. Scale bar: $20 \mu \mathrm{m}$. Dashed lines delineate gut villi (A.), mature spermatid area (B.), or kidney tubules (C.). At 9 months of age, no differences in apoptosis were detected in gut, testis and KM comparing tert-/- No Cre, tert-/- +Cre and WT fish All data are represented as mean $+/-$ SEM $(\mathrm{N}=5-7$ per condition; no significance was detected comparing all conditions and using one-way ANOVA and post-hoc Tuckey tests). 

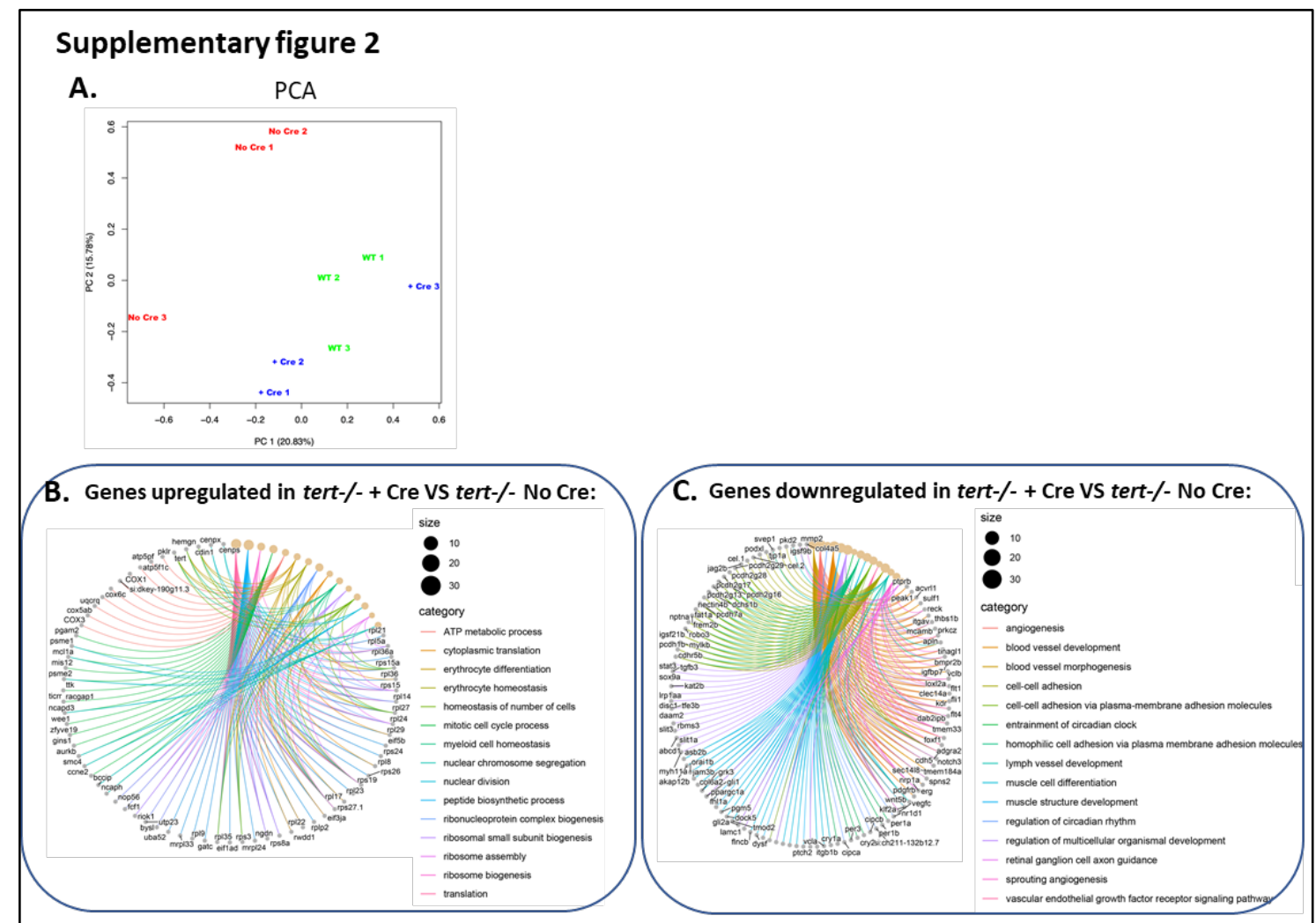

D. Genes upregulated in WT VS tert-/- No Cre:

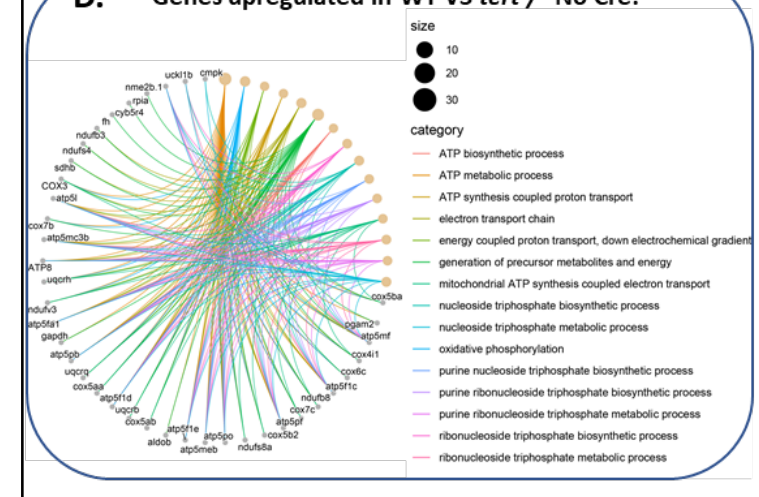

E. Genes downregulated in WT VS tert-/- No Cre:

F.

Ribosome

Oxidative phosphorylation

Adrenergic signaling in cardiomyocytes

Phagosome

Endocytosis

Apelin signaling pathway

Autophagy - animal

Insulin signaling pathway

Calcium signaling pathway

MAPK signaling pathway

Cytokine-cytokine receptor interaction

Wnt signaling pathway

Vascular smooth muscle contraction

Focal adhesion

Neuroactive ligand-receptor interaction

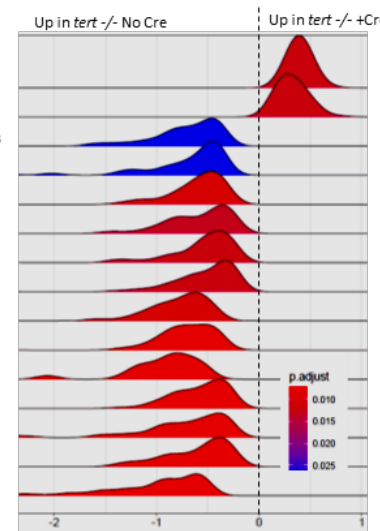

G.

Ribosome

Oxidative phosphorylation

Biosynthesis of cofactors

Endocytosis

Cytokine-cytokine receptor interaction

Lysosome

Wnt signaling pathway

MAPK signaling pathway

Phagosome

Calcium signaling pathway

Neuroactive ligand-receptor interaction Focal adhesion

Supplementary figure 2: Gut specific tert expression rescues gut transcriptomic profile.

A. Principal Component Analysis (PCA) based on untargeted transcriptomic data of 9-month-old gut samples. A clustering between tert-/- +Cre and WT while tert-/- No Cre group was clearly distinguishable from tert-/- No Cre fish (N=3 per group). B-C. Identification of Differentially Expressed Genes (DEGs) and their biological process categories (Gene Ontology -GO- Term analysis; FDR $<0.1$ ) that are downregulated (B.) or upregulated (C.) in the gut of 9-month-old tert-/- +Cre fish compared to tert-/- No Cre fish. D-E. Identification of 
bioRxiv preprint doi: https://doi.org/10.1101/2022.01.10.475664; this version posted January 11,2022 . The copyright holder for this preprint (which was not certified by peer review) is the author/funder, who has granted bioRxiv a license to display the preprint in perpetuity. It is made available under aCC-BY-ND 4.0 International license.

that are downregulated (D.) or upregulated (E.) in the gut of 9-month-old WT fish compared to tert-/- No Cre fish. Genes associated with morphogenesis, angiogenesis, cell-cell adhesion and muscle development are concomitantly downregulated compared to tert-/- No Cre reflecting the requirement of tissue repair in tert-/- No Cre fish. In parallel, ATP metabolism, ribonucleotide biosynthesis and mitotic cell cycle processes pathways are enriched in tert-/- +Cre and WT fish compared to tert-/- No Cre suggesting mitochondrial defects and reduced proliferation in tert-/- No Cre fish. F-G. Identification of KEGG (Kyoto Encyclopedia of Genes and Genomes) term using GSEA (Gene Set Enrichment Analysis) in the gut of 9-month-old tert-/- +Cre (F.) or WT (G.) fish compared to tert-/- No Cre. Genes associated with Ribosome and Oxidative phosphorylation pathways are enriched in the gut of both tert-/- +Cre and WT fish reflecting higher translation process and mitochondrial function compared to tert-/- No Cre. KEGG terms related to Cytokine-cytokine receptor interaction, Phagosome, Endocytosis, Neuroactive ligand-receptor interaction, Focal adhesion, Wnt signaling, MAPK signaling pathway and Calcium signaling pathway are reduced in both tert-/- +Cre and WT fish compared to tert-/- No Cre suggesting higher inflammation and requirement for tissue repair mechanisms.

\section{Supplementary figure 3}

A. c_Alphaproteobacteria
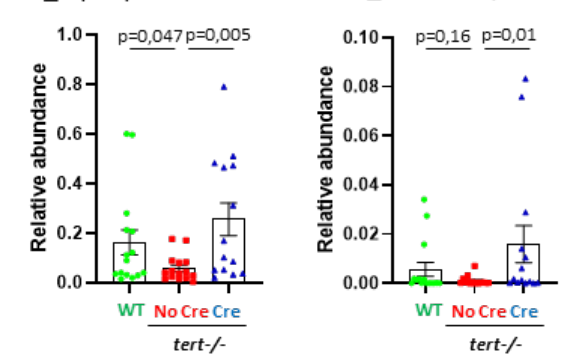

B.
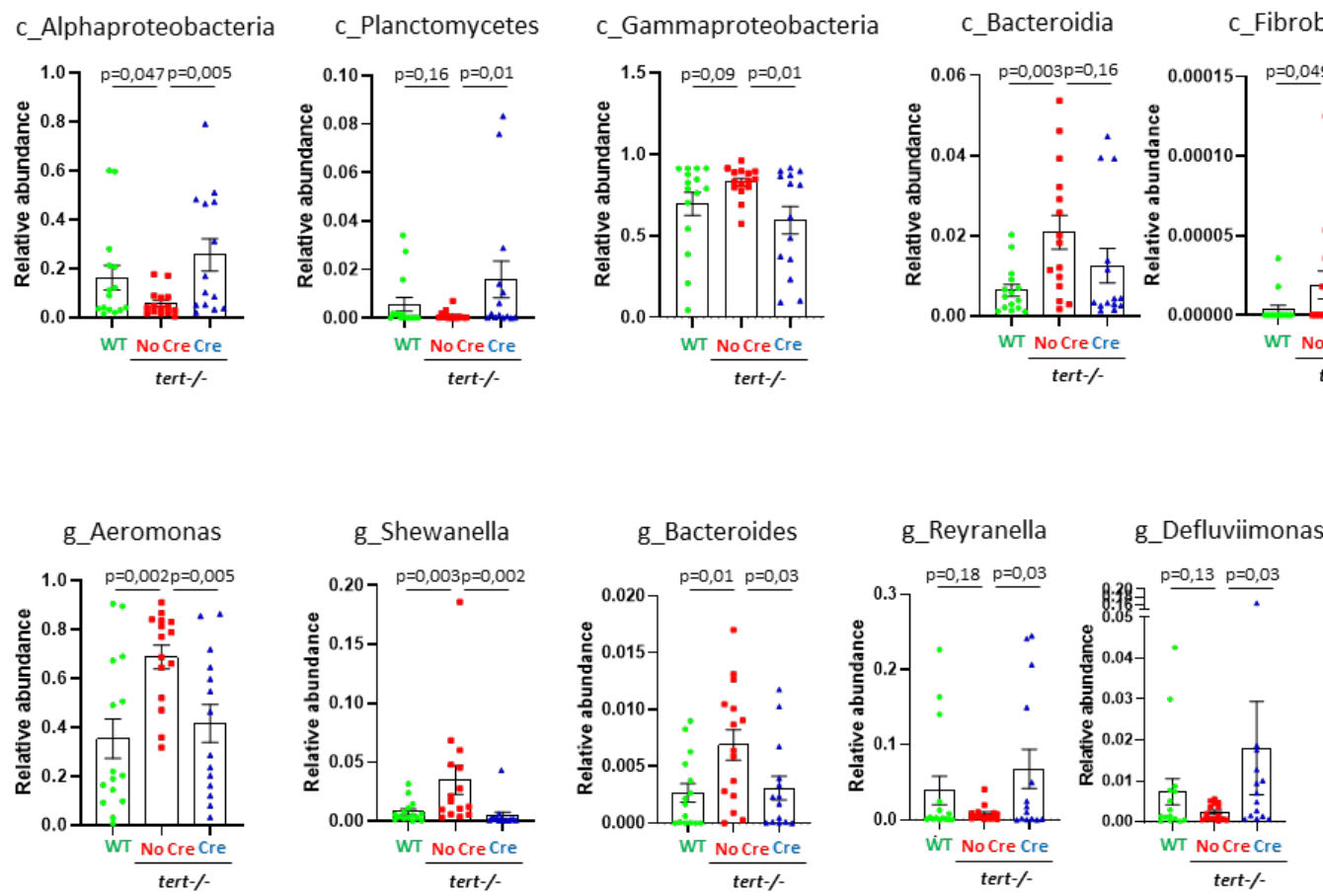

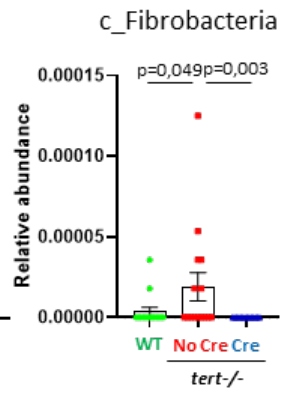

C. s_Aeromonas_veronii
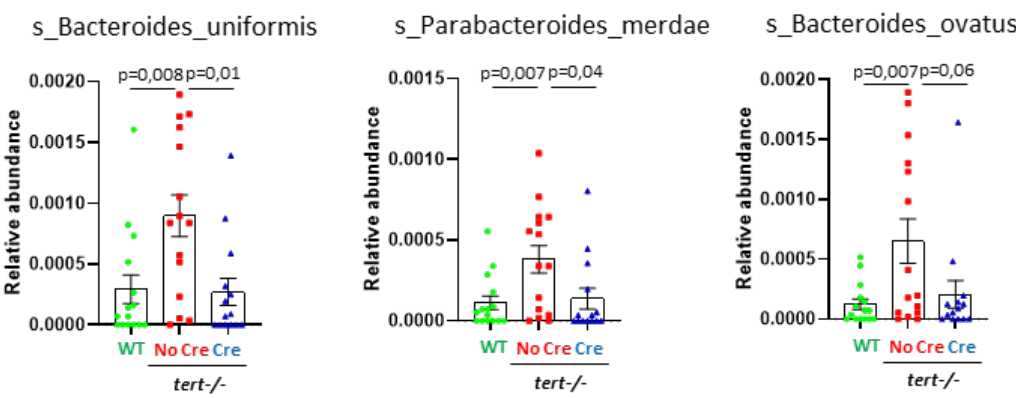

\section{Supplementary figure 3: Gut-specific telomerase activity rescues alterations of gut microbiota composition.}

A-C. Relative abundance analysis of bacteria at the level of class (A.); genus (B.) and species (C.). $\mathrm{N}=14-15$; $\mathrm{p}$ values were determined using Multiple hypothesis-test for sparsely-sampled features and false discovery rate (FDR). Tert expression in gut of tert-/- fish (tert-/- +Cre) recapitulates bacteria abundance at the class and species levels to WT profile compared to tert-/- No Cre where pathogenic bacteria are enriched. 


\section{Supplementary figure 4}

A. Downregulated metabolites

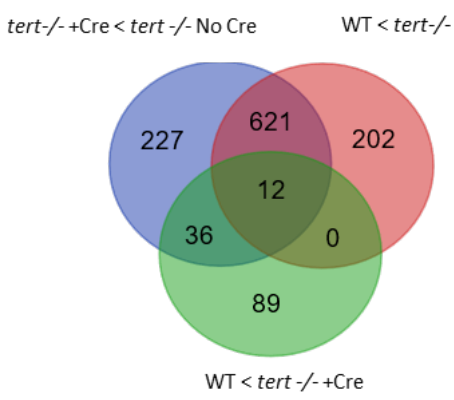

\section{Upregulated metabolites}

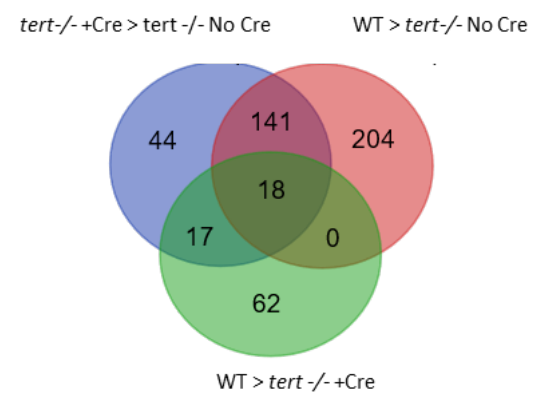

B. Anaerobic glycolysis:

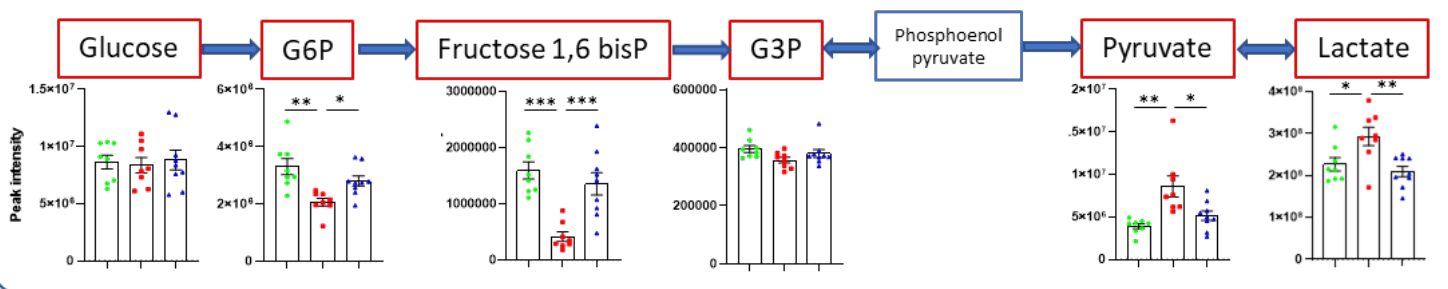

C.

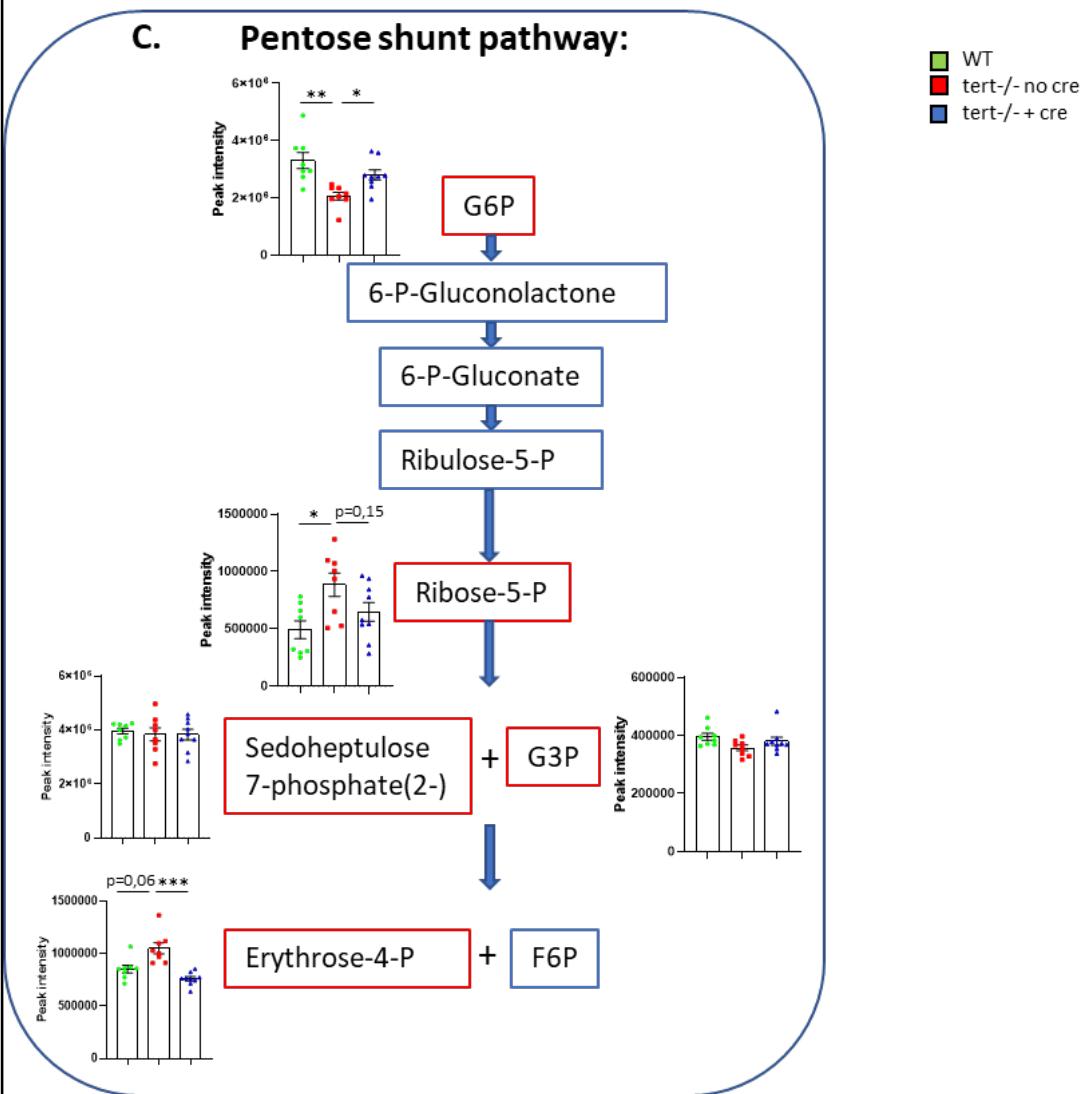

무

Supplementary figure 4: Gut-specific telomerase activity rescues gut metabolomic

\section{profile.}

A. Venn diagram representing downregulated (left panel) or upregulated (right panel) metabolites comparing the three conditions in gut of 9-month-old fish. The majority of metabolites detected in the gut of 9 months-old fish are concomitantly down or up-regulated in tert-/- +Cre and WT groups compared to tert-/- No Cre fish. B-C. 
bioRxiv preprint doi: https://doi.org/10.1101/2022.01.10.475664; this version posted January 11,2022 . The copyright holder for this preprint (which was not certified by peer review) is the author/funder, who has granted bioRxiv a license to display the preprint in perpetuity. It is made available under aCC-BY-ND 4.0 International license.

Supplementary figure 4 continued: fish. Anaerobic glycolysis and pentose shunt metabolic profiles are rescued to WT levels in the gut of tert-/+ Cre compared tert-/- No Cre fish. All data are represented as mean +/- SEM (N=8-9 per condition; * $\mathrm{p}-$ value $<0.05$; ** $p$-value $<0.01, * * *$ p-value $<0.001$, using one-way ANOVA and post-hoc Tuckey tests). Red squares: detected metabolites; blue squares: undetected metabolites.

\section{Supplementary figure 5}

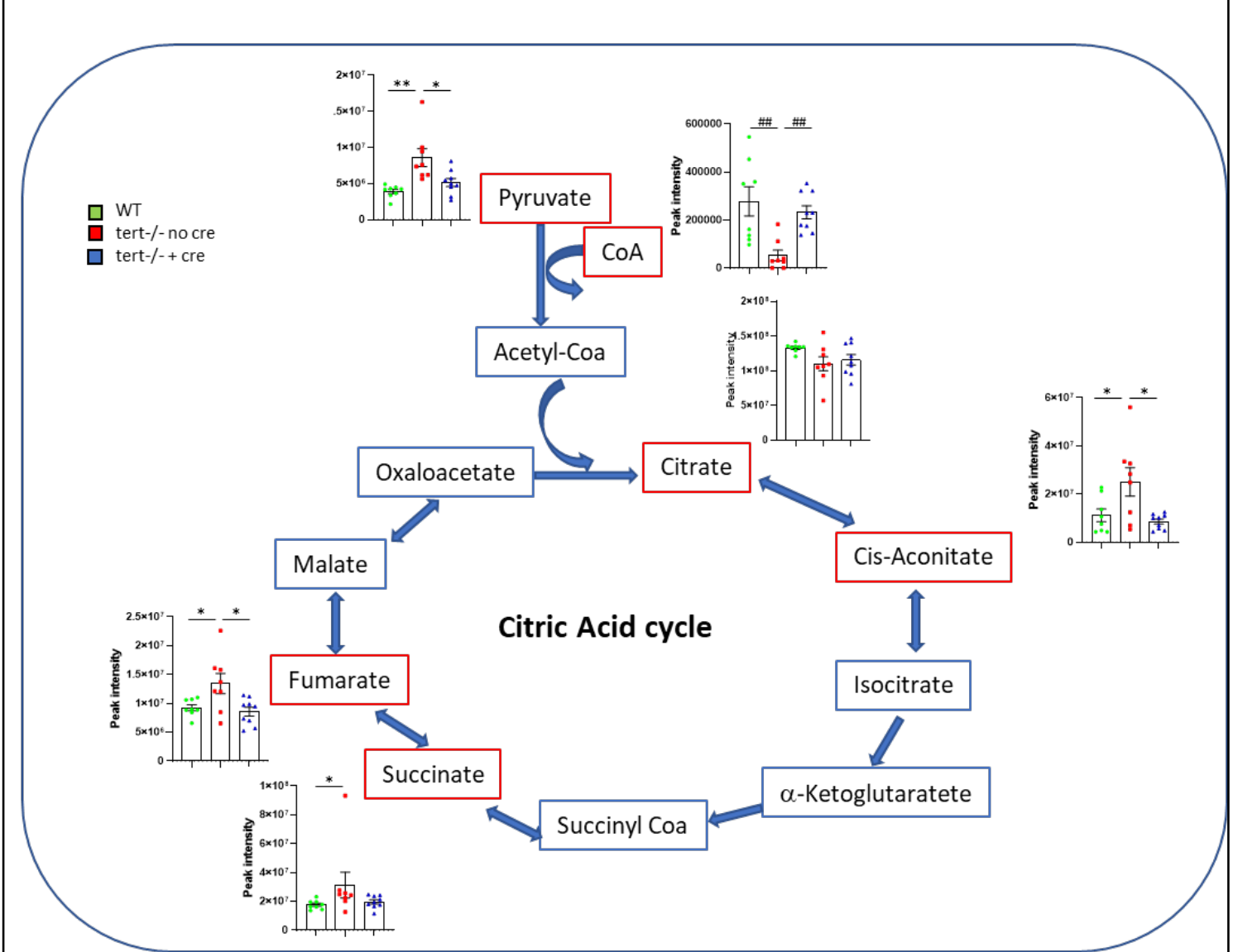

Supplementary figure 5: Gut-specific telomerase activity rescues citric acid cycle metabolism alterations in the gut of tert-/- fish.

Metabolomic analysis of the citric acid cycle gut of 9-month-old fish. Citric cycle metabolic profile in the gut of tert-/- +Cre is similar to WT compared tert-/- No Cre fish. All data are represented as mean +/- SEM (N=8-9 per condition; * p-value $<0.05 ; * *$ p-value $<0.01$, using one-way ANOVA and post-hoc Tuckey tests; \#\# pvalue $<0.01$, using Kruskal-Wallis and post-hoc Dunn's tests). Red squares: detected metabolites; blue squares: 


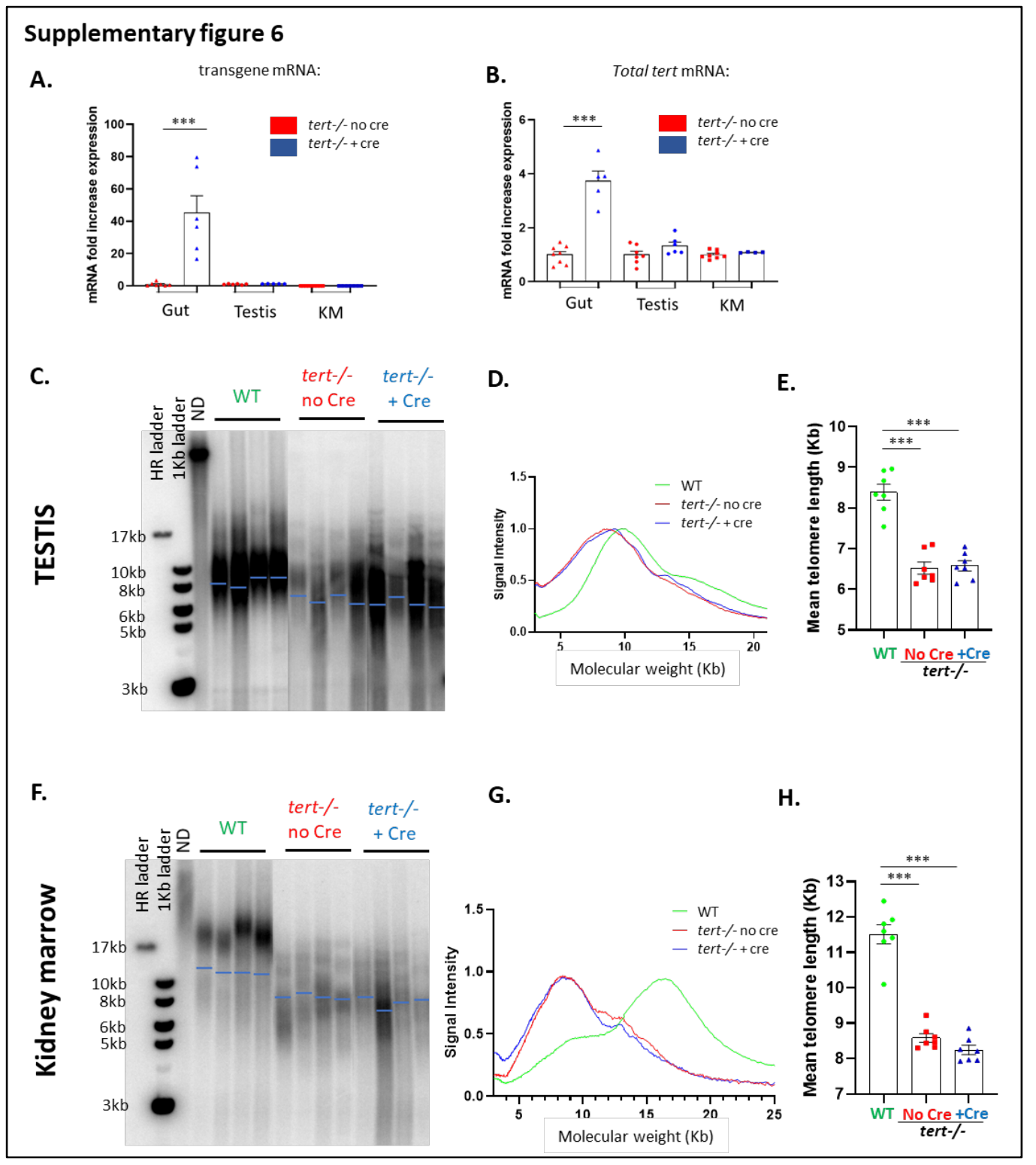

Supplementary figure 6: Cre-mediated tert transgene expression is specific to gut tissue. expression in gut, testis of KM extracts from 9-month-old tert-/- No Cre and tert-/- +Cre fish. RT-qPCR graphs are representing mean \pm SEM mRNA fold increase after normalization by $r p s 11$ gene expression levels $(\mathrm{N}=5-8$; $* * *$ p-value $<0.001$, using one-way ANOVA and post-hoc Tuckey tests). While tert transgene transcription is induced by Cre injection in the gut of tert-/- fish compared to tert-/- No Cre, no transgene expression was detected in testis and KM of tert-/ + Cre fish. C. Representative images of telomere restriction fragment (TRF) analysis by Southern Blot of genomic DNA extracted from 9-month-old testis samples and quantifications of mean telomere length (blue bars). D. TRF mean densitometry curves from 9-month-old testis samples $(\mathrm{N}=6-7)$. E. Quantification of mean telomere length analyzed by TRF on testis from 9-month-old fish. Data are represented as mean $+/$ - SEM ( $\mathrm{N}=6-7 ; * * *$ p-value $<0.001$, using one-way ANOVA and post-hoc Tuckey tests). F. Representative images of telomere restriction fragment (TRF) analysis by Southern Blot of genomic DNA extracted from 9-month-old KM samples and quantifications of mean telomere length (blue bars). G. TRF mean densitometry curves from 9-month-old $\mathrm{KM}$ samples $(\mathrm{N}=6-7)$. H. Quantification of mean telomere length analyzed by TRF on KM from 9-month-old fish. Data are represented as mean +/- SEM (N=6-7; *** pvalue $<0.001$, using one-way ANOVA and post-hoc Tuckey tests). In accordance with lack of transgene expression in testis and $\mathrm{KM}$, no difference in telomere length was detected in these organs when comparing tert- 


\section{Bibliography:}

825

826

827

828

829

830

831

832

833

834

835

836

837

838

839

840

841

842

843

844

845

846

847

848

849

850

851

852

853

854

855

856

857

858

859

860

861

862

863

864

865

866

867

868

869

870

871

1. Kenyon, C., Chang, J., Gensch, E., Rudner, A. \& Tabtiang, R. A C. elegans mutant that lives twice as long as wild type. Nature 366, 461-4 (1993).

2. López-Otín, C., Blasco, M. A., Partridge, L., Serrano, M. \& Kroemer, G. The Hallmarks of Aging. Cell 153, 1194-1217 (2013).

3. Kenyon, C. J. The genetics of ageing. Nature 464, 504-512 (2010).

4. Funk, M. C., Zhou, J. \& Boutros, M. Ageing, metabolism and the intestine. EMBO Rep. 21, e50047 (2020).

5. Kirkland, J. L. \& Tchkonia, T. Senolytic drugs: from discovery to translation. J. Intern. Med. 288, 518-536 (2020).

6. Baker, D. J. et al. Clearance of p16Ink4a-positive senescent cells delays ageingassociated disorders. Nature 479, 232-6 (2011).

7. Blackburn, E. H., Greider, C. W. \& Szostak, J. W. Telomeres and telomerase: the path from maize, Tetrahymena and yeast to human cancer and aging. Nat. Med. 12, 1133-8 (2006).

8. Shay, J. W. \& Wright, W. E. Hayflick, his limit, and cellular ageing. Nat. Rev. Mol. Cell Biol. 1, 72-76 (2000).

9. Blackburn, E. H. \& Francisco, S. Telomeres. Encycl. LIFE Sci. 1-7 (2001).

10. Yui, J., Chiu, C. P. \& Lansdorp, P. M. Telomerase activity in candidate stem cells from fetal liver and adult bone marrow. Blood 91, 3255-3262 (1998).

11. Artandi, S. E. \& DePinho, R. a. Telomeres and telomerase in cancer. Carcinogenesis 31, 9-18 (2009).

12. Mitchell, J. R., Wood, E. \& Collins, K. A telomerase component is defective in the human disease dyskeratosis congenita. Nature 402, 551-5 (1999).

13. Opresko, P. L. \& Shay, J. W. Telomere-associated aging disorders. Ageing Res. Rev. 33, 52-66 (2017).

14. Carneiro, M. C. et al. Short Telomeres in Key Tissues Initiate Local and Systemic Aging in Zebrafish. PLoS Genet. 12, (2016).

15. Henriques, C. M., Carneiro, M. C., Tenente, I. M., Jacinto, A. \& Ferreira, M. G. Telomerase is required for zebrafish lifespan. PLoS Genet. 9, e1003214 (2013).

16. Anchelin, M. et al. Premature aging in telomerase-deficient zebrafish. Dis. Model. Mech. 6, 1101-12 (2013).

17. E1 Maï, M., Marzullo, M., de Castro, I. P. \& Ferreira, M. G. Opposing p53 and $\mathrm{mTOR} / \mathrm{AKT}$ promote an in vivo switch from apoptosis to senescence upon telomere shortening in zebrafish. Elife 9, 1-26 (2020).

18. Demanelis, K. et al. Determinants of telomere length across human tissues. Science 369, (2020).

19. Jonassaint, N. L., Guo, N., Califano, J. A., Montgomery, E. A. \& Armanios, M. The gastrointestinal manifestations of telomere-mediated disease. Aging Cell 12, 319-23 (2013).

20. Glousker, G., Touzot, F., Revy, P., Tzfati, Y. \& Savage, S. A. Unraveling the pathogenesis of Hoyeraal-Hreidarsson syndrome, a complex telomere biology disorder. Br. J. Haematol. 170, 457-71 (2015).

21. Kinouchi, Y. et al. Telomere shortening in the colonic mucosa of patients with ulcerative colitis. J. Gastroenterol. 33, 343-8 (1998).

22. Risques, R. A. et al. Ulcerative colitis is a disease of accelerated colon aging: evidence from telomere attrition and DNA damage. Gastroenterology 135, 410-8 (2008).

23. Biagi, E. et al. Through ageing, and beyond: gut microbiota and inflammatory status in 
seniors and centenarians. PLoS One 5, e10667 (2010).

24. O'Toole, P. W. \& Jeffery, I. B. Gut microbiota and aging. Science 350, 1214-5 (2015).

25. Thevaranjan, N. et al. Age-Associated Microbial Dysbiosis Promotes Intestinal Permeability, Systemic Inflammation, and Macrophage Dysfunction. Cell Host Microbe 21, 455-466.e4 (2017).

26. Kanther, M. et al. Microbial colonization induces dynamic temporal and spatial patterns of NF- $\kappa \mathrm{B}$ activation in the zebrafish digestive tract. Gastroenterology 141, 197-207 (2011).

27. Ma, Y.-C. et al. YAP in epithelium senses gut barrier loss to deploy defenses against pathogens. PLOS Pathog. 16, e1008766 (2020).

28. Gregorieff, A., Liu, Y., Inanlou, M. R., Khomchuk, Y.\& Wrana, J. L. Yap-dependent reprogramming of Lgr5+ stem cells drives intestinal regeneration and cancer. Nature 526, 715-718 (2015).

29. Tran, L. \& Greenwood-Van Meerveld, B. Age-associated remodeling of the intestinal epithelial barrier. Journals Gerontol. - Ser. A Biol. Sci. Med. Sci. 68, 1045-1056 (2013).

30. Raju, P. et al. Inactivation of paracellular cation-selective claudin-2 channels attenuates immune-mediated experimental colitis in mice. J. Clin. Invest. 130, 5197-5208 (2020).

31. Clark, R. I. et al. Distinct Shifts in Microbiota Composition during Drosophila Aging Impair Intestinal Function and Drive Mortality. Cell Rep. 12, 1656-67 (2015).

32. Claesson, M. J. et al. Gut microbiota composition correlates with diet and health in the elderly. Nature 488, 178-84 (2012).

33. Batut, J., Andersson, S. G. E. \& O'Callaghan, D. The evolution of chronic infection strategies in the $\alpha$-proteobacteria. Nat. Rev. Microbiol. 2, 933-945 (2004).

34. Huang, Y. T. et al. Genomic and phylogenetic characterization of Shewanella xiamenensis isolated from giant grouper (Epinephelus lanceolatus) in Taiwan. Zoonoses Public Health 66, 679-685 (2019).

35. Mukhopadhya, I. et al. A comprehensive evaluation of colonic mucosal isolates of sutterella wadsworthensis from inflammatory bowel disease. PLoS One 6, 1-10 (2011).

36. Hiippala, K., Kainulainen, V., Kalliomäki, M., Arkkila, P. \& Satokari, R. Mucosal prevalence and interactions with the epithelium indicate commensalism of Sutterella spp. Front. Microbiol. 7, 1-13 (2016).

37. Round, J. L. \& Mazmanian, S. K. The gut microbiota shapes intestinal immune responses during health and disease. Nat. Rev. Immunol. 9, 313-323 (2009).

38. Saitoh, S. et al. Bacteroides ovatus as the predominant commensal intestinal microbe causing a systemic antibody response in inflammatory bowel disease. Clin. Diagn. Lab. Immunol. 9, 54-59 (2002).

39. Kenny, H. A. et al. Quantitative high throughput screening using a primary human three-dimensional organotypic culture predicts in vivo efficacy. Nat. Commun. 6, 6220 (2015).

40. López-Otín, C., Galluzzi, L., Freije, J. M. P., Madeo, F. \& Kroemer, G. Metabolic Control of Longevity. Cell 166, 802-821 (2016).

41. Srivastava, S. Emerging insights into the metabolic alterations in aging using metabolomics. Metabolites 9, 1-16 (2019).

42. Rudolph, K. L. et al. Longevity, stress response, and cancer in aging telomerasedeficient mice. Cell 96, 701-712 (1999).

43. Townsley, D. M., Dumitriu, B. \& Young, N. S. Bone marrow failure and the telomeropathies. Blood 124, 2775-2783 (2014).

44. Thongon, N. et al. Hematopoiesis under telomere attrition at the single-cell resolution. Nat. Commun. 12, (2021). 
45. Jaskelioff, M. et al. Telomerase reactivation reverses tissue degeneration in aged telomerase-deficient mice. Nature 469, 102-106 (2011).

46. Tomás-Loba, A. et al. Telomerase Reverse Transcriptase Delays Aging in CancerResistant Mice. Cell 135, 609-622 (2008).

47. Chakravarti, D. et al. Telomere dysfunction activates YAP1 to drive tissue inflammation. Nat. Commun. 11, 4766 (2020).

48. Esteves, A. et al. Fatty acid binding proteins have the potential to channel dietary fatty acids into enterocyte nuclei. J. Lipid Res. 57, 219-232 (2016).

49. Chin, L. et al. P53 Deficiency Rescues the Adverse Effects of Telomere Loss and Cooperates With Telomere Dysfunction To Accelerate Carcinogenesis. Cell 97, 527538 (1999).

50. Paramos-de-Carvalho, D., Jacinto, A. \& Saúde, L. The right time for senescence. Elife 10, 139-141 (2021).

51. Campisi, J. Aging, cellular senescence, and cancer. Annu. Rev. Physiol. 75, 685-705 (2013).

52. Fan, Y. \& Pedersen, O. Gut microbiota in human metabolic health and disease. Nat. Rev. Microbiol. 19, 55-71 (2021).

53. Smith, P. et al. Regulation of life span by the gut microbiota in the short-lived African turquoise killifish. Elife 6, 120980 (2017).

54. Ostrakhovitch, E. A. \& Tabibzadeh, S. Homocysteine and age-associated disorders. Ageing Res. Rev. 49, 144-164 (2019).

55. Bárcena, C. et al. Methionine Restriction Extends Lifespan in Progeroid Mice and Alters Lipid and Bile Acid Metabolism. Cell Rep. 24, 2392-2403 (2018).

56. Kitada, M., Ogura, Y., Monno, I., Xu, J. \& Koya, D. Effect of methionine restriction on aging: Its relationship to oxidative stress. Biomedicines 9, 1-15 (2021).

57. Imamura, S. et al. A non-canonical function of zebrafish telomerase reverse transcriptase is required for developmental hematopoiesis. PLoS One 3, (2008).

58. Mosimann, C. et al. Ubiquitous transgene expression and Cre-based recombination driven by the ubiquitin promoter in zebrafish. Development 138, 169-177 (2011).

59. Caporaso, J. G. et al. Ultra-high-throughput microbial community analysis on the Illumina HiSeq and MiSeq platforms. ISME J. 6, 1621-1624 (2012). 